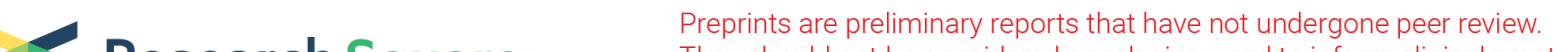 Research Square
Thr ef should not be considered conclusive, used to inform clinical practice,
of the media as validated information.
}

\section{Excitatory drive of cortical fast-spiking GABAergic interneurons is set by D-serine acting on NMDA receptors}

\section{Pierre Lecouflet}

1Université Paris-Saclay, CNRS, ENS Paris-Saclay, CentraleSupélec, Laboratoire Lumière, Matière et Interfaces (LuMin) UMR9024

\section{Steeve Maldera}

1Université Paris-Saclay, CNRS, ENS Paris-Saclay, CentraleSupélec, Laboratoire Lumière, Matière et Interfaces (LuMin) UMR9024

\section{Brigitte Potier}

1Université Paris-Saclay, CNRS, ENS Paris-Saclay, CentraleSupélec, Laboratoire Lumière, Matière et Interfaces (LuMin) UMR9024

\section{Loredano Pollegioni}

University of Insubria https://orcid.org/0000-0003-1733-7243

Jean-Pierre Mothet ( $\nabla$ jean-pierre.mothet@universite-paris-saclay.fr)

1 Université Paris-Saclay, CNRS, ENS Paris-Saclay, CentraleSupélec, Laboratoire Lumière, Matière et Interfaces (LuMin) UMR9024 https://orcid.org/0000-0003-4991-0614

\section{Article}

Keywords:

Posted Date: February 4th, 2022

DOI: https://doi.org/10.21203/rs.3.rs-1323674/v1

License: (c) (i) This work is licensed under a Creative Commons Attribution 4.0 International License. Read Full License 


\section{Abstract}

$\mathrm{N}$-methyl-D-aspartate receptors (NMDARs) populate GABAergic interneurons, where they play a critical role in shaping circuit motifs and memory. However, we are largely ignoring whether and how NMDARs at GABAergic interneurons are gated by signals released in their surrounding microenvironment. Here we explore the dynamics of the co-agonist site occupancy by D-serine and glycine at glutamatergic synapses onto parvalbumin positive GABAergic interneurons in the adolescent prefrontal cortex, an area central to complex cognitive operation. We discovered that $\mathrm{D}$-serine but not glycine is required for maintaining the activity of NMDAR at the GABAergic interneurons and that the identity of the co-agonist is not determined by the synaptic regime. Our study extends the physiological implications of D-serine in brain physiopathology by uncovering its control of inhibitory synaptic networks through NMDARs.

\section{Introduction}

GABAergic interneurons are in charge of the dynamic adjustment of the level of excitation insured by glutamatergic neurons, in maintaining a proper excitation/inhibition balance, and are therefore essential for information processing within the brain ${ }^{1,2}$. The fast-spiking parvalbumin-containing $\left(\mathrm{FS}^{-} \mathrm{PV}^{+}\right)$neurons represent the largest population of cortical inhibitory neurons ${ }^{1}$ and by providing the major somatic inhibitory drive to excitatory pyramidal cells (PCs) play a critical role in circuit physiology ${ }^{3,4}$. As a consequence, malfunction of these GABAergic interneurons is central to several neuropsychiatric disorders $^{5-7}$.

FS- $\mathrm{PV}^{+}$interneurons are primarily recruited by glutamatergic synaptic input from the PCs. In particular, $\mathrm{FS}^{-\mathrm{PV}^{+}}$interneurons express $\mathrm{N}$-methyl-D-aspartate receptors (NMDARs) that are pivotal for circuit entrainment and for generating network oscillations that encode numerous cognitive processes. Selective ablation of NMDARs during early postnatal development results in synaptic dysconnectivity with PCs and in corrupted network oscillations and behavioural deficits ${ }^{8-10}$. Thus, hypofunction of NMDAR onto PV ${ }^{+}$ interneurons has emerged as a cause of various neurodevelopmental disorders including schizophrenia (SCZ) and autism spectrum disorders (ASD) ${ }^{10,11}$. However, how NMDAR at FS-PV ${ }^{+}$are physiologically regulated by extracellular signals is unknown. NMDARs are heterotetrameric molecular devices typically composed of two GluN1 subunits and two GluN2 subunits ${ }^{12,13}$. Yet, activation of the canonical NMDARs requires simultaneous binding of I-glutamate and a co-agonist, glycine or d-serine ${ }^{12-14}$. Paradoxically, more than two decades after the discovery of the action of glycine and d-serine at NMDAR ${ }^{15-17}$, we are still ignoring how the functions of GABAergic interneurons are regulated by NMDAR co-agonists and the physiopathological relevance of such modulation remains unaddressed. In this study, we examined the potential contributions of d-serine and glycine in controlling the activity and functions of NMDARs at FS$\mathrm{PV}^{+}$interneurons in the prelimbic area (PrL) of the prefrontal cortex (PFC), a brain area central to complex

cognitive operation ${ }^{18,19}$ which dysfunction is invariably implicated in the physiopathology of $\mathrm{SCZ}^{20}$ and then explored the physiological conditions that specify their actions. By combining cellular electrophysiology with the use of unique pharmacological interventions and genetic manipulations, we 
report that $\mathrm{d}$-serine is the sole co-agonist controlling NMDAR at FS-PV ${ }^{+}$interneurons under different synaptic activity levels. We further show that $d$-serine-deficient mice, a model of NMDAR hypofunction that exhibits schizophrenia-like phenotypes display attenuated firing pattern of the interneurons and no long-term potentiation. These results provide new insights on the cell-type dependent mode of regulation of NMDAR by their co-agonists. They also widen the physiological landscape of $d$-serine in brain functioning opening new opportunities for precise therapeutic interventions.

\section{Results}

Functional NMDA receptors controls intrinsic excitability of FS-PV ${ }^{+}$interneurons. We focused on the regulation of FS-PV ${ }^{+}$interneurons of the layer 5 of PrL that can be readily identified in $\mathrm{PV}^{+}$-tdTomato fluorescent reporter mice (Fig. 1a) during late adolescence which is a critical timing period for the onset of clinical symptoms for many psychiatric disorders in humans ${ }^{21}$. Upon injection of depolarizing current pulses, these interneurons exhibited typical firing patterns of high frequency and non-adaptive spikes (Supplementary Fig. 1) with short lasting action potentials (APs) (half-width $\sim 0.51 \pm 0.06 \mathrm{~ms}$ ) and large fast after-hyperpolarization (AHPs, $21.48 \pm 4.03 \mathrm{mV}$ ). Although functional NMDARs are largely populating different classes of GABAergic interneurons ${ }^{22,23}$ early during cortical development, their presence in adolescent and adult FS-PV ${ }^{+}$interneurons remains debated ${ }^{24}$. To probe for the presence of functional NMDARs at FS-PV ${ }^{+}$interneurons, we first analyzed the current-voltage (I-V) relationship of isolated excitatory postsynaptic currents (EPSCs) from the interneurons of layer 5 of PrL (Supplementary Fig. 2a, b). NMDA-EPSCs were pharmacologically isolated using blockers of a-amino-3-hydroxy-5-methyl-4isoxazolepropionic acid (AMPA) receptors((2,3-dioxo-6-nitro-1,2,3,4-tetrahydrobenzo[f]quinoxaline-7-sul-

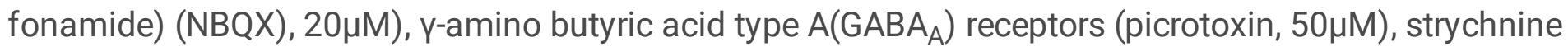
sensitive glycine receptors (strychnine, $10 \mu \mathrm{M})$ and in normal $\mathrm{Mg}^{2+}(1.5 \mathrm{mM})$. Electrical stimulation $(0.1$ $\mathrm{Hz}$ ) elicited typical I-V curve of NMDA-EPSCs (Supplementary Fig. $2 \mathrm{~b}$ ) that reversed at $0 \mathrm{mV}$ and showed inward rectifications. Inclusion of the non-competitive NMDAR antagonist (5R,10S)-(+)-5-methyl-10,11dihydro-5H-dibenzo[a,d]-cyclohepten-5-10-imine (MK801; 2-3 mM) in the intracellular solution of the recording pipette (iMK801) ${ }^{25,26}$ selectively blocked NMDA-EPSCs (Supplementary Fig. 2b, c) while preserving the AMPA-EPSCs (Supplementary Fig. 2c). Noteworthy, the NMDAR/AMPAR peak amplitude ratio was $\sim 1.0$ indicating that FS-PV ${ }^{+}$interneurons in late adolescent mice still express large fraction of operational NMDARs. We then investigated whether NMDARs could control intrinsic FS-PV ${ }^{+}$bursting activity and be tonically activated. Inclusion of MK801 in the recording pipette consistently reduced the excitability of FS-PV ${ }^{+}$neurons (Fig. 1b; Supplementary Fig. 3) supporting the idea that NMDARs of $\mathrm{PV}^{+}$ neurons are tonically activated. Application of NMDA $(10 \mu \mathrm{M})$, consistently increased the firing activity of FS-PV ${ }^{+}$interneurons (Supplementary Fig. 2d, e and Supplementary Fig. 3), an effect systematically nulled by iMK801. Therefore, operational NMDARs largely populate FS-PV ${ }^{+}$interneurons beyond early postnatal development ${ }^{27}$ where they significantly contribute to modulate the firing and synaptic activities of the inhibitory interneurons. 


\section{Regulation of NMDA receptors by d-serine selectively controls the intrinsic excitability of FS-PV ${ }^{+}$}

interneurons. We next interrogated whether the bursting activity of $\mathrm{FS}^{-\mathrm{PV}^{+}}$neurons might be determined by the level of the NMDAR co-agonist site occupancy. Increasing glycine levels by blocking the glycine transporter GlyT1 with N-[(3R)-3-([1,1'-biphenyl]-4-yloxy)-3-(4-fluorophenyl)propyl]-N-methylglycine hydrochloride (ALX5407; $1 \mu \mathrm{M})^{28-31}$ did not alter spike frequency of FS-PV+ neurons (Fig. 1c). Conversely, favoring the presence of endogenous $d$-serine by bath-application of CBIO $(1 \mu \mathrm{M})^{31-33}$ an inhibitor of the d-serine degrading enzyme D-amino acid oxidase (DAAO) $)^{14,34-37}$ increased the activity of FS-PV ${ }^{+}$neurons (Fig. 1d; Supplementary Fig. 3) but remarkedly not of PCs (Supplementary Fig. 4). Inclusion of MK801 (2$3 \mathrm{mM}$ ) in the intracellular recording pipette impeded the potentiating action of CBIO (Fig. 1e) thus demonstrating that the modulation of $\mathrm{FS}^{-} \mathrm{PV}^{+}$interneurons activity does not result from network activity and is most likely caused by a direct action of $d$-serine acting on the NMDARs at the GABAergic neurons. In the brain, $d$-serine is synthesized by serine racemase ${ }^{14,30,31,38-40}$. We then explored the intrinsic firing properties of FS-PV+ interneurons from SR-null mutant mice $\left(\mathrm{SR}^{-/}\right)$which are deficient in d-serine ${ }^{41-45}$. Strikingly, $\mathrm{FS}^{-\mathrm{PV}^{+}}$interneurons from $\mathrm{SR}^{-/-}$mice showed a marked reduction in their intrinsic excitability in comparison to wild-type (WT) littermate mice (Fig. 1f; Supplementary Fig. 3). Altogether, our findings support the view that the activity of the GABAergic FS-PV ${ }^{+}$interneurons highly depends on the occupancy of the NMDAR co-agonist binding site by d-serine and that these NMDAR are tonically activated under basal conditions.

\section{Co-agonism by d-serine but not glycine selectively controls NMDA-EPSCs in interneurons. Since the level} of occupancy of the co-agonist site of NMDAR by d-serine is critical for driving $\mathrm{FS}^{-\mathrm{PV}^{+}}$interneurons firing activity, we hypothesized that it could also determine their synaptic coupling with PCs. We then evaluated the physiological consequences of such modulations by recording NMDA-EPSCs from L5 FS-PV ${ }^{+}$cells (Fig. 2). As before, NMDA-EPSCs were pharmacologically isolated using NBQX $(20 \mu \mathrm{M})$, picrotoxin $(50 \mu \mathrm{M})$ and strychnine $(10 \mu \mathrm{M})$. To further precise the identity of the co-agonists in controlling the activity of NMDAR at cortical GABAergic interneurons, we used enzymatic scavengers that specifically blunt the action of endogenous d-serine or glycine by degrading them in the extracellular space. Selective and acute depletion of d-serine with $R g D A A O(0.2 \mathrm{U} / \mathrm{mL})^{29-31,46}$ did not affect the voltage-activity dependency of the NMDA-EPSCs (Fig. 2a) but yielded to a 50\% reduction in the NMDA/AMPA ratio tied to its specific eroding action on NMDA-EPSCs (Fig. 2b). Analysis of the paired-pulse ratio (PPR) of NMDAEPSCs showed no difference between control and RgDAAO groups (Supplementary Fig. 5) thus confirming that $d$-serine acts specifically on postsynaptic NMDARs expressed by FS-PV ${ }^{+}$cells. As shown in Fig. 2b, NMDA-EPSCs persisted in the presence of RgDAAO suggesting that glycine which is left intact in these experiments might also contribute to regulate synaptic NMDARs ${ }^{29-31}$. The putative role of glycine in the persistent d-AP5 sensitive NMDA-EPSCs in the presence of RgDAAO is excluded by addition of $B s G O(0.2 \mathrm{U} / \mathrm{mL})$ that selectively degrades glycine ${ }^{29-31}$ but did not further impact the amplitude of NMDA-EPSCs (Fig. 2b). As expected, CBIO that elevates synaptic levels of d-serine increased NMDAEPSCs (Fig. 2c) suggesting that the coagonist site of synaptic NMDAR on FS-PV ${ }^{+}$cells was not saturated by the ambient levels of the endogenous co-agonist. Intriguingly, blockade of GlyT1 with ALX5407 that 
increases glycine levels increased the amplitude of NMDA-EPSCs as well (Fig. 2c). Therefore, rendering glycine available at the synapse enables NMDAR modulation but is not sufficient to influence the firing activity (Fig. 1C). CV-2 analysis revealed that CBIO and ALX5407 exerted their potentiating effects at purely postsynaptic NMDARs (Supplementary Fig. 6). In conclusion, these results revealed that NMDAR at FS-PV ${ }^{+}$interneurons in adolescence are primarily gated by d-serine and not glycine at low activity regime and point that glycine transporters (e.g GlyT1) are efficiently maintaining the levels of glycine below effective concentrations within the synaptic cleft as already observed at excitatory synapses between PCs in the hippocampus ${ }^{29,31}$ or amygdala ${ }^{30}$.

\section{Selective loss-of-function of d-serine impairs synaptic plasticity of PC excitatory output synapse onto FS-}

$\mathrm{PV}^{+}$cells. We next explored whether the identity of the co-agonist driving NMDAR at $\mathrm{PV}^{+}$interneurons might be determined by the level of synaptic activity ${ }^{30,31}$. To do so, we analyzed the role of the co-agonist in controlling NMDAR-dependent short- and long-term excitatory synaptic plasticity at the PC to FS-PV ${ }^{+}$ connections (Fig. 3a, b). To study short-term plasticity, we examined the effects of realistic trains of stimulation of the synapse at low physiological 2,20 or $50 \mathrm{~Hz}$ trains typical of $\mathrm{PFC} \mathrm{PV}^{+}$cells firing rates during attentional processing ${ }^{47-49}$. At 2 and $20 \mathrm{~Hz}$ individual NMDA-EPSCs were clearly discernible for each pulse of stimulus current and exhibit amplitude attenuation which was precipitated by increasing frequencies ${ }^{50,51}$ (Fig. 3a). Acute d-serine depletion by treating the slices with RgDAAO accelerated and increased the depression rate at 2 and $20 \mathrm{~Hz}$ in comparison to control slices. At 50Hz, NMDAR-EPSCs were not discernible and exhibited temporal summation of the first three trains before substantial depression appeared (Fig. 3a). Such pattern is most likely related to the slow kinetics of the NMDAR channels ${ }^{12,13}$ thus occluding single NMDA-EPSCs to return to baseline before the next stimulation. Anyway, no difference was observed at $50 \mathrm{~Hz}$ between the RgDAAO treated and control groups indicating that the synaptic depression became independent of the levels of the co-agonist at $y$ ranges. Most importantly, these data show that the presence of a threshold level of d-serine is necessary for NMDAR to maintain persistent states of activity at theta and beta frequencies $(\sim 2-20 \mathrm{~Hz})$.

Although excitatory output synapses onto $\mathrm{FS}^{-\mathrm{PV}^{+}}$interneurons have been shown to undergo various forms of activity-dependent long term plasticity ${ }^{52-57}$ whether NMDAR-dependent long-term potentiation (LTP) of excitatory synapses onto GABAergic interneurons would then rely on a specific co-agonist is still unknown. Hebbian NMDAR-dependent LTP requires coincident glutamate release and postsynaptic depolarization to relieve channel blockade by $\mathrm{Mg}^{2+}$ ions. We therefore tested a Hebbian protocol consisting in pairing afferent tetanus high-frequency stimulation (HFS) with postsynaptic depolarization of the FS-PV ${ }^{+}$cells to $0 \mathrm{mV}$. This protocol invariably induced a D-AP5 sensitive long-term potentiation (LTP) of the excitatory input synapse onto FS-PV ${ }^{+}$interneurons (Fig. 3b) that appeared not to depend on somatodendritic voltage-gated $\mathrm{Na}^{+}$channels activation since $5 \mathrm{mM}$ QX314 was included in the whole-cell patch pipette. The mean paired pulse ratio (PPR) of evoked EPSCs at $30 \mathrm{~ms}$ apart was not significantly changed upon this Hebbian LTP at FS-PV ${ }^{+}$cells (Supplementary Fig. 7a). In addition, analysis of $\mathrm{CV}^{-2}$ confirmed the postsynaptic locus of the LTP (Supplementary Fig. 7b). These results suggest that this 
form of LTP is expressed with no apparent change in presynaptic function (e.g transmitter release) and further support that it does not involve presynaptic NMDARs. More importantly, the same protocol failed to induce LTP in the presence of RgDAAO thus phenocopying the blocking action of D-AP5 (Fig. 3b). In conclusion, excitatory input synapses onto $\mathrm{FS}^{-} \mathrm{PV}^{+}$neurons can undergo homosynaptic LTP that relies exclusively on the activation of postsynaptic NMDAR by d-serine.

\section{NMDA-EPSCs at FS-PV ${ }^{+}$interneurons are not altered in a genetic model of NMDAR hypofunction. To} confirm the pivotal role of $d$-serine, we next anticipated that the activity of NMDAR at FS-PV ${ }^{+}$interneurons would be ultimately affected in the $\mathrm{SR}^{-/-}$mice (Fig. 4a), a translational model of NMDAR

hypofunction ${ }^{41,42,44,58,59}$. Unexpectedly, no significant differences were found in the voltage dependency and peak amplitude of NMDA-EPSCs recorded from FS-PV ${ }^{+}$neurons in $\mathrm{SR}^{-/-} \mathrm{PFC}$ (Fig. 4b, c). In addition, NMDA/AMPA ratio was unaffected in $\mathrm{SR}^{-/}$slices when compared to WT slices (Fig. 4c). However, we noticed that NMDA-EPSCs from FS-PV ${ }^{+}$cells in $\mathrm{SR}^{-/-}$mice systematically displayed a longer rising time (Fig. 4d) but no change in decay kinetics. As rise-time of EPSCs reflects the opening rate of the NMDAR channel, we envision that the mode of activation of the NMDARs in $\mathrm{SR}^{-/-}$mice might likely differ from WT mice because of increased dwell time of neuromodulatory signals in the extracellular space. We then explored the underlying mechanisms that could contribute to maintain fully functional NMDAR in $\mathrm{SR}^{-/-}$ mice in the absence of $d$-serine and that could result in slowering rise-time. We had previously found that BsGO did not affect NMDA-EPSCs in WT slices (Fig. 2b). Conversely, addition of $B s G O(0.2 \mathrm{U} / \mathrm{mL})$ to $\mathrm{SR}^{-/}$ slices remarkably reduced the NMDA-EPSCs by $~ 60 \%$ without altering AMPA-EPSCs ending in a net decrease in the NMDA/AMPA ratio (Fig. 4c). These data demonstrate that glycine could serve as a spare co-agonist for synaptic NMDARs at excitatory synapses onto GABAergic interneurons but solely in the chronic absence of $d$-serine. Building on these observations, we inferred that the slowing kinetics of risetime of NMDA-EPSCs is a consequence of increased glycine spillover and increased dwell time of the coagonist in the extracellular space consequent to the reduced uptake of glycine by astrocytes in $\mathrm{SR}^{-/-}$mice rather than a change in glutamate residency in the synaptic cleft.

\section{Co-agonism by glycine incompletely rescues the absence of $d$-serine during synaptic plasticity. We then} wondered whether this compensation by glycine in $\mathrm{SR}^{-/-}$mice we observed during basal synaptic transmission could fully meet or not the needs for NMDAR functioning when increasing the synaptic regime. As shown above, at $50 \mathrm{~Hz}$ the synaptic attenuation and summation does not depend anymore on the degree of occupancy of the NMDAR co-agonist binding site, we thus restricted our evaluation of NMDA-EPSCs to 2 and $20 \mathrm{~Hz}$ in PFC slices isolated from SR ${ }^{-1}$. Interestingly, we found no difference with control WT littermates at $2 \mathrm{~Hz}$ but revealed that short-term plasticity in $\mathrm{SR}^{-/}$slices was altered at $20 \mathrm{~Hz}$ (Fig. 5a). These results thus indicated that glycine could sustain NMDAR activity at low firing rate below $20 \mathrm{~Hz}$ but beyond, the system became invariably unstable in the absence of $d$-serine. Finally, we considered the potential contribution of glycine in enabling LTP of the PC to FS-PV ${ }^{+}$excitatory synaptic contacts in the $\mathrm{SR}^{-/-}$mice. As previously reported, HFS invariably induced NMDAR-dependent LTP in WT mice but this protocol was ineffective in triggering LTP in $\mathrm{SR}^{-/-}$mice (Fig. 5b). This effect was not 
associated with changes in presynaptic parameters (Supplementary Fig. 7c, d) but most likely reflect a loss of neuromodulation of postsynaptic NMDARs. Therefore glycine, contrary to d-serine and despite its action on NMDARs at low synaptic regime, is not engaged in the formation of LTP at excitatory synapses onto FS-PV ${ }^{+}$interneurons. Overall these results argue that glycine could compensate for the chronic absence of d-serine (e.g., $\mathrm{SR}^{-/}$) to support the demand of NMDAR activity only at low but not at high synaptic regimes in striking contrast to reported observations gathered on synapses between excitatory neurons ${ }^{30,46,60,61}$.

\section{Discussion}

One key conclusion from the present study is that d-serine but not glycine modulates the excitatory drive of cortical GABAergic $\mathrm{PV}^{+}$interneurons by acting on NMDAR. We demonstrate that specific limited functions of d-serine induce NMDAR hypofunction impairing burst firing and long term synaptic plasticity. Here, inhibitory neurons were targeted using PV- tdTomato reporter mice and then no distinction between the chandelier and basket cells types neurons was made. However, even if both cell populations are present in the mouse mPFC, only the basket cells are found in deep layers ${ }^{62}$. Furthermore, the unique fastspiking electrophysiological features of the $\mathrm{PV}^{+}$neurons we recorded here indicate that we are most likely indeed dealing with basket cells.

Although d-serine (and also glycine) could bind to the glutamate insensitive diheteromeric GluN1-GluN3 and homotetrameric GluD receptors ${ }^{12,13}$ that populate excitatory and inhibitory neurons, the modulatory actions of $d$-serine (and to a lesser extend of glycine) at $\mathrm{PV}^{+}$interneurons we are now reporting are not mediated by these unconventional ionotropic glutamate receptors since all elicited responses were blocked by the non-competitive antagonists d-AP5 or MK801. Furthermore, the analysis of current-voltage $(\mathrm{I}-\mathrm{V})$ curves of NMDA-EPSCs recorded at $\mathrm{PV}^{+}$interneurons show voltage-dependent rectifications typical of GluN1-GluN2 conventional receptors in normal $\mathrm{Mg}^{2+}(1.5 \mathrm{mM})$. Nevertheless, the subtypes of GluN1GluN2 NMDAR involved in these modulations by the co-agonist remains uncertain and need to be defined in future studies.

Two recent studies have reported that $\mathrm{SR}^{-/-}$mice have reduced inhibitory tone in cortical networks resulting in corrupted neural synchrony and $E / I$ balance ${ }^{60,61}$, suggesting a critical role of d-Ser in regulating the activity of GABAergic interneurons. However, the specific cell population of interneurons types and mechanisms remain largely unclear. In addition, so far, studies have explored the relative roles of the co-agonists at afferent excitatory synapses onto pyramidal neurons in different brain areas ${ }^{29-}$ 31,41,42,46,63-67 but none have addressed their roles in controlling the activity of GABAergic interneurons. These studies concluded that, i) both glycine and d-serine cooperate to regulate NMDAR functions at the excitatory PC-to-PC synapses, ii) the identity of the co-agonist is determined by the level of synaptic activity $29-31,46,63$, and iii) under pathological conditions of chronic d-serine absence as occurring in the $\mathrm{SR}^{-/-}$mice, glycine can fully replace d-serine and support the activity of NMDARs at excitatory 
neurons $\mathrm{s}^{30,60,61}$ especially in the $\mathrm{mPFC}{ }^{46}$. Owing to the fact that $\mathrm{SR}^{-/-}$mice displayed impaired gamma oscillations ${ }^{58}$ and the critical roles of $\mathrm{PV}^{+}$interneurons in circuit's dynamics and their key implication in SCZ pathogenesis as the putative cell locus of NMDAR hypofunction, we decided to focus on this celltype. Here, we probed the level of activation of the NMDAR co-agonist site at FS-PV ${ }^{+}$interneurons by endogenously released coagonist under different patterns of synaptic activity and pharmacological interventions. We found that the site is unsaturated in physiological conditions which is consistent with the notion that the basal concentration of endogenous co-agonist is insufficient to saturate the NMDAR and that enhancing its recruitment can improve the output functions of the GABAergic neurons. We show that the identity of the co-agonist (eg d-serine) at GABAergic neurons is not dictated by the level of afferent excitatory inputs activity and that glycine can only sparely replace $d$-serine. Indeed, compensation by glycine of NMDAR gating in $\mathrm{SR}^{-/}$mice or under acute loss-of function of $\mathrm{d}$-serine (e.g $R g \mathrm{DAAO})$ is incomplete and deficits of excitatory drive of $\mathrm{PV}^{+}$interneurons already appear at low beta frequency range $(\sim 20 \mathrm{~Hz})$. Since glutamate is fully saturating NMDARs during synaptic transmission ${ }^{68}, \mathrm{~d}-$ serine availability is the limiting factor for optimal NMDAR activation at $\mathrm{PV}^{+}$interneurons. Accordingly, despite normal synaptic transmission at low synaptic regimes $(<2 \mathrm{~Hz})$, HFS-induced LTP is occluded in $\mathrm{SR}^{-/}$mice just phenocopying the effect of $R g \mathrm{DAAO}$ treatment. Although here we used a HFS to induce LTP in the presence of the GABAR blocker picrotoxin and strychnine to disable unwanted feed-forward inhibition as a minimal experimental paradigm, it is tempting to speculate that even using bursts repeated at the theta-frequency $(\sim 5 \mathrm{~Hz})$ would have not recruited glycine and would have fail to rescue LTP. Indeed, a recent study reported that $\mathrm{SR}^{-1-}$ mice had deficits in frontal cortex evoked power across the beta $(20-30 \mathrm{~Hz})$ and the gamma $(30-80 \mathrm{~Hz})$ frequencies ${ }^{58}$. A role for glycine in wild-type mice (e.g when dserine is present) was unmasked only when measuring NMDA-EPSCs after blocking the GlyT1 with ALX5407 (Fig. 2) which seems at odds with the non-implication of the amino acid in the control of burst firing of $\mathrm{PV}^{+}$cells. This apparent discrepancy could be related to the fact that bursting activities are recorded at the resting membrane potential under current clamp thus better preserving the physiological conditions. NMDA synaptic currents were collected at $+40 \mathrm{mV}$ to relieve the potential-dependent $\mathrm{Mg}^{2+}$ block of the channel and in response to non-minimal perisomatic stimulations. These experimental conditions are prompted to place the recorded cell and its local environment in an upper 'non physiological' excitatory state that may have unsilenced some NMDARs normally non active at physiological membrane potentials and thus may favor the engagement of glycine. Henceforth, it is tempting to envision the existence of at least two populations of NMDARs that are differently distributed along the somatodendritic arbor of the $\mathrm{PV}^{+}$interneurons that would be accessible for gating by d-serine and to a lesser degree by glycine depending on the physiological needs. We also show that the effect of blocking GlyT1 with ALX5407 is greater than the effect of inhibiting DAAO with CBIO on NMDA-EPSCs amplitude. Although, the mechanisms of inhibition is different (transporter versus an enzyme) and the pharmacological effects may relate to kinetics of GlyT1 vs DAAO, these results predict that the levels of $d$ serine in the synaptic cleft are near saturation while the levels of glycine are insufficient for the normal activation of NMDARs. Altogether, by reporting that only pharmacological or genetic-induced manipulations of d-serine levels did alter the firing activities of the $\mathrm{PV}^{+}$cells in a NMDAR-dependent 
manner, we demonstrate that $d$-serine but not glycine is the permissive factor engaged in the tonic control of NMDAR in physiological conditions.

The mechanisms underlying the selective action of $d$-serine at $\mathrm{PV}^{+}$inhibitory neurons remain to be addressed. Although the NMDARs subunits composition may differ between excitatory and inhibitory neurons ${ }^{12}$, both neuromodulators display nearly similar sensitivity to NMDARs independently of their subunits composition ${ }^{12,13}$. The partial compensatory action of glycine at GABAergic neurons in $\mathrm{SR}^{-/-}$mice may rather reflect differences in the dynamics and availability of the co-agonists between excitatory synapses onto principal cells $v s$ inhibitory neurons, as already observed for glutamate ${ }^{69}$. Astrocytes by expressing the GlyT1 are critically involved in regulating the ambient levels of glycine at the proximity of excitatory neurons ${ }^{29-31}$. Hence, the synaptic microenvironment of GABAergic and glutamatergic neurons would be largely influenced by the morphological interposition of individual synapse with astrocytes ${ }^{69-}$ 71. Yet, excitatory neurons establish close contact with astrocytes leaflets while GABAergic neurons notably the $\mathrm{PV}^{+}$interneurons establish sparse contact with glia ${ }^{71}$ although still contributing to inhibitory synaptic signalization. On line with these observations, pharmacological blockade of GlyT1 by ALX5407 to build up ambient glycine levels did not alter the firing activity of GABAergic interneurons while inhibition of DAAO by CBIO to boost $d$-serine synaptic availability positively increases firing activity of $\mathrm{PV}^{+}$interneurons and their synaptic coupling with PCs. Accordingly, it is tempting to speculate that $\mathrm{d}-$ serine which behave as the strict co-agonist for NMDAR at $\mathrm{PV}^{+}$interneurons would be preferentially formed by the postsynaptic GABAergic neurons themselves or by their apposed presynaptic glutamatergic partner without much involving glia. Delineation of the precise role(s) of these respective partners in the dynamics and synaptic disposition of d-serine (and glycine) in inhibitory network signalization has to be determined in future studies. The presence of active DAAO in the forebrain has been questioned for decades and therefore its role in d-serine catabolism remains an opened issue ${ }^{34-36}$. Our previous work indicated that the potentiating effect of CBIO on excitatory synaptic transmission in the hippocampus circuitry was nulled in $\mathrm{SR}^{-/}$mice thus demonstrating that the effect of the compound by inhibiting DAAO was caused by an elevation in $\mathrm{d}$-serine ${ }^{31}$. Here, our pharmacological experiments using $\mathrm{CBIO}$ further evidence that DAAO is present in the forebrain and also is the enzyme involved in $\mathrm{d}$ serine catabolism but most importantly, show that DAAO by controlling d-serine disposition play a critical role in controlling the activity of GABAergic interneurons as well,

Importantly, in addition to unearth NMDAR hypofunction at $\mathrm{PV}^{+}$interneurons in $\mathrm{SR}^{--}$mice, our results indicate how NMDAR hypofunction impairs PV neuronal functions by demonstrating impaired burst firing. Burst firing of $\mathrm{PV}^{+}$interneurons promote the reliability of somatic inhibitory drive to PCs thus playing a cardinal role in the temporal precision of local pyramidal firing and in the generation of coherent oscillations enabling the synchronization of large PC populations and proper behaviors ${ }^{1-10}$. Recent findings suggest that NMDARs in PV interneurons enhances the probability of GABA release in the $\mathrm{mPFC}^{26}$. Therefore, we predict that the presence of persistent deprived burst firing in PV neurons as observed in $\mathrm{SR}^{--}$mice would result in abnormal PC disinhibition and alter PV-to-PV cross-inhibition during 
behavioral tasks that fine-regulate local oscillations. We have recently reported that the firing activity of layer $5 \mathrm{PCs}$ in the mPFC of adolescent mice is unaffected in $\mathrm{SR}^{-/-}$mice ${ }^{46}$. Therefore, our new data further support the idea that the co-agonist site of NMDAR at the PV+ GABAergic neurons is more sensitive to $d$ serine withdrawal as compared to PCs. Accordingly, we further highlight that the level of occupancy of the NMDAR co-agonist binding site depends on the cell type reflecting how synapses on PCs and on interneurons adopt different morphology and functional properties ${ }^{69-71}$. Altogether, our observations thus offer a mechanistic explanation why $\mathrm{SR}^{-/-}$mice show impairments in gamma oscillations and social interactions ${ }^{58,59}$.

Lastly, besides transforming our vision of brain circuit's physiology, our study is of main clinical relevance by fully justifying current therapeutics strategies targeting the co-agonist site of NMDARs by d-serine in the management of $\mathrm{SCZ}$ and others brain disorders ${ }^{11}$. Indeed, NMDAR hypofunction at $\mathrm{PV}^{+}$interneurons has been invariably proposed to play a pivotal role in the pathogenesis of many psychiatric disorders, such as $S C Z^{5,11}$ and studies suggest that these neurons should be considered as viable targets to more efficient antipsychotics. Yet, DAAO inhibitors are seen as one of the most promising pharmacological approach to counteract the cognitive and negative symptoms caused by NMDAR hypofunction ${ }^{72}$. Our study connects these different emerging concepts by reporting that loss-of-function of $d$-serine induces selective NMDAR hypofunction at $\mathrm{PV}^{+}$interneurons and that $\mathrm{DAAO}$ inhibition with $\mathrm{CBIO}$ increases firing activity of $\mathrm{PV}^{+}$interneurons but not of $\mathrm{PCs}$. Although $\mathrm{PV}^{+}$interneurons may be central to NMDAR hypofunction in SCZ, dysfunctions are likely spreading to other inhibitory cell-types including the SST and CCK neurons since the latter are expressing NMDAR ${ }^{22,23}$ as well. Additional studies would be needed to elucidate the roles of the co-agonists at these different cell populations and the physiopathological relevance of these modulations.

In summary, our study sheds new light on brain circuit's physiology by uncovering the general principles of NMDARs regulation at GABAergic interneurons by upstream signals released in their surrounding microenvironment. Building on these findings, we identify that $\mathrm{d}$-serine is critical for network computations by engaging NMDARs of $\mathrm{PV}^{+}$interneurons and that loss of its functions would recapitulate the synaptic deficits in disease like SCZ and could be exploited for the future development of more effective clinical interventions targeting inhibitory neurons.

\section{Methods}

Animals. Experiments were performed on 40 to 60 day-old (mean $=54 \pm 10$ days) male and female mice (with corresponding control littermates) from four different lines: C57BL/6, serine racemase knock-out $\left(\mathrm{SR}^{-/-}\right)$mice (C57BL/6 genetic background) ${ }^{41}$ and the transgenic parvalbumin( $\left.\mathrm{PV}^{+}\right)$-tdTomato mice (Jackson Laboratories, stock no 027395), which express the red fluorescent protein tdTomato in PVpositive interneurons (C57BL/6 genetic background) ${ }^{73}$. $\mathrm{SR}^{-/-}$and $\mathrm{SR}^{+/+}$mice were crossed with $\mathrm{PV}^{+}-$ tdTomato animals in order to obtain double transgenic mice for ready identification of $\mathrm{PV}^{+}$-cells with either SR genotype. Only Tg/Tg::SR ${ }^{+/+}$or $\mathrm{Tg} / \mathrm{Tg}:: \mathrm{SR}^{-/-}$were used in the study. In some cases, to identify 
$\mathrm{PV}^{+}$neurons, we used Ai9-tdTomato:PV-Cre transgenic mice generated from crossing PV-IRES-Cre knockin mice (Jackson Laboratories, stock no. 008069) with Ai9-tdTomato reporter knock-mice (Jackson Laboratories, stock no. 007905).

Animals were housed in groups in polycarbonate cages and maintained on a 12/12 hr light/dark cycle (light on at $7 \mathrm{am})$ in a temperature $\left(22^{\circ} \mathrm{C}\right)$ and humidity controlled room. Animals were given access to food and water ad libitum. All experiments performed in France complied with the European Union recommendations (2010/63/EU) and were approved by the French Ministry of Agriculture and Fisheries (authorization number authorization No. 75 1273) and by the University Paris-Saclay (accreditation number C92-019-01).

Slice preparation. Animals were deeply anesthetized with isoflurane before cervical dislocation and decapitation. The brain was then quickly removed and placed in ice-cold slicing artificial cerebrospinal fluid (ACSF) containing (in mM): $\mathrm{KCl} 3.5, \mathrm{MgSO}_{4} 7, \mathrm{CaCl}_{2}$ 0.5, D-glucose 11, sucrose 212, $\mathrm{NaH}_{2} \mathrm{PO}_{4} 1.2$, $\mathrm{NaHCO}_{3}$ 26.2, L-ascorbic acid 1.2 and Na-pyruvate 2.4 (pH 7.3-7.4 and 302-313 mOsml/L), continuously bubbled with $95 \% \mathrm{O}_{2} / 5 \% \mathrm{CO}_{2}$. Coronal slices of the prefrontal cortex ( $350 \mu \mathrm{m}$ thickness) were then prepared similarly for all mice lines using a vibrating blade microtome and allowed to recover in regular ACSF containing (in mM): $\mathrm{NaCl} 124, \mathrm{KCl} 3.5, \mathrm{MgSO}_{4} 1.5, \mathrm{CaCl}_{2} 2.5$, D-glucose $11, \mathrm{NaH}_{2} \mathrm{PO}_{4} 1.2, \mathrm{NaHCO}_{3}$ 26.2, saturated with $95 \% \mathrm{O}_{2} / 5 \% \mathrm{CO}_{2}(\mathrm{pH} 7.4,325 \mathrm{mOsml} / \mathrm{L})$ during $30 \mathrm{~min}$ at $32^{\circ} \mathrm{C}$ and for additional 30 min at room temperature before recordings.

Brain slices electrophysiology and data analysis. Slices were transferred to a recording chamber mounted either on a upright Slicescope Pro 6000 system (Scientifica, UK) and continuously perfused with oxygenated ACSF $(2-3 \mathrm{~mL} / \mathrm{min})$ at near physiological temperature $\left(30^{\circ}-32^{\circ} \mathrm{C}\right.$, Heated perfusion tube HPT2A, ALA Scientific Instruments Inc, USA). Recordings were performed from FS-PV ${ }^{+}$neurons located in layer 5 of the prelimbic region (PrL) of the medial PFC. Cells were visualized by infrared differential interference contrast and with a cooled digital CCD OrcaR ${ }^{2}$ Hamamatsu camera (C10600-10B, Hamamatsu Photonics, Japan) in combination with an on-line video contrast enhancement. Epifluorescence illumination (CoolLED pE-2 excitation system) was used to visualize tdTomato-positive interneurons (Ex/Em: 555/581nm), using differential interference contrast infrared videomicroscopy.

Recordings were obtained using a Multiclamp 700B amplifier (Molecular devices, CA, USA) and signals were filtered at $2 \mathrm{kHz}$ and digitized at $5 \mathrm{kHz}$ via a DigiData 1440A interface (Molecular Devices, CA, USA). Data were collected and analyzed using pClamp10 software (Molecular Devices, CA, USA). Series resistance and holding current were monitored throughout the experiment. Cells with an access resistance $>25 \mathrm{M} \Omega$ at resting potential were excluded from analyses as well as any cell for which $a>20 \%$ change in those parameters occurred during the course of the experiment.

Electrophysiological recording were made using borosilicate glass pipettes (resistance $=3-5 \mathrm{M} \Omega$ ) filled with either one of the two following intracellular solutions, depending on the experiment : K-gluconate solution (in mM: K-gluconate 120, KCl 10, HEPES 10, EGTA 0.2, MgATP 4.5, NaGTP 0.3 and Na- 
phosphocreatine 14, pH 7.2-7.3;290-295 mOsm/L) or Cs-methanesulfonate solution (in mM: $\mathrm{CsCH}_{4} \mathrm{O}_{3} \mathrm{~S}$ 140, $\mathrm{CsCl} 6, \mathrm{MgCl}_{2}$ 2, HEPES 10, EGTA 1,1, QX-314 5 and MgATP 4, pH 7.2-7.3 ; 295-300 mOsm/L). Neurons were first clamped at $-70 \mathrm{mV}$ and allowed to dialyze for $5 \mathrm{~min}$ before any recording. All recordings were done in the presence of strychnine $(10 \mu \mathrm{M})$ and picrotoxin $(50 \mu \mathrm{M})$ in the bathing ACSF to block glycinergic and $\mathrm{GABA}_{A}$ receptors, respectively. All drugs needed for control conditions were applied 10 minutes before recording and during the full length of the experiment. Subsequent drug effects were measured 5 minutes after a plateau was obtained and compared to baseline. In some experiments, MK801 was added to the intracellular solution to block NMDAR at the recorded cell.

Membrane and firing properties of $\mathrm{PC}$ and $\mathrm{FS}_{-} \mathrm{PV}^{+}$neurons were recorded in current-clamp mode using Kgluconate solution including or not MK801 (iMK801: 2-3 mM). Results were obtained in response of square step current injections (500 msec) into the recorded cell from -100 pA to 200 pA with 20 pA steps. Spike frequency adaptation was measured as the ratio of the first inter-spike interval duration over the 8th one at rheobase $+40 \mathrm{pA}$.

Evoked postsynaptic excitatory (EPSCs) currents were recorded in voltage-clamp mode and elicited by focal extracellular stimulation using bipolar tungsten electrodes positioned at $50 \mu \mathrm{m}$ from the recording site and connected to a stimulus isolation unit (World Precision Instruments, FL, USA) at a frequency of $0.1 \mathrm{~Hz}$ unless specified otherwise. Evoked AMPA EPSCs were recorded from FS-PV ${ }^{+}$neurons clamped at $-70 \mathrm{mV}$ in the presence of PTX $(50 \mu \mathrm{M})$ and strychnine $(10 \mu \mathrm{M})$ using Cs-methanesulfonate intrapipette solution with or without MK801 (2-3 mM). NMDA EPSCs were isolated by maintaining FS-PV ${ }^{+}$neurons at $+40 \mathrm{mV}$ in the presence of NBQX $(20 \mu \mathrm{M})$ in the perfusion medium. The NMDAR/AMPAR ratio of the EPSCs was calculated as the ratio of NMDA-EPSC at $+40 \mathrm{mV}$ and AMPA-EPSC at $-70 \mathrm{mV}$. I-V relationship of NMDA-EPSCs was measured at holding potentials from $-70 \mathrm{mV}$ to $+40 \mathrm{mV}$ with $10 \mathrm{mV}$ steps and amplitude was normalized as a fraction of the amplitude at $+40 \mathrm{mV}$.

For synaptic plasticity experiments, $\mathrm{FS}-\mathrm{PV}^{+}$neurons were recorded with the K-gluconate internal solution with QX-314 included when assessing long-term potentiation (LTP) to prevent firing of action potentials during induction ${ }^{74}$. Short term plasticity was investigated by two means: paired-pulse ratios (PPR, interstimulus intervals (ISI) ranging from 50 to $250 \mathrm{~ms}$ ) and trains of 20 stimulations at varying frequencies (2-50 Hz). LTP was investigated by maintaining the postsynaptic FS-PV ${ }^{+}$cell at $-70 \mathrm{mV}$ during a 5 minutes baseline then at $0 \mathrm{mV}$ during the conditioning stimulus. Afterward, the holding potential was set back at $-70 \mathrm{mV}$ for a $30 \mathrm{~min}$ post-conditioning recording. Excitatory LTP was elicited by high frequency tetanus (1 $\mathrm{sec}$ at $100 \mathrm{~Hz}$ repeated twice at a $20 \mathrm{sec}$ interval). PPR was recorded before baseline and $30 \mathrm{~min}$ after LTP induction to inquire about the pre- versus post-synaptic locus of expression of the LTP. ISI was 30 ms for EPSCs.

To further precise the locus of action of the drugs, the coefficient of variation (CV) analysis method was used $^{26}$. The proportional change in the inverse square of the coefficient of variation $\left(\mathrm{CV}^{-2}\right)$ was compared with the proportional change in the mean postsynaptic potential amplitude $(\mathrm{M})$ to determine whether the 
quantal amplitude (q), the release probability (p) or the number of release sites (n) had changed. In a binomial distribution, $\mathrm{CV}^{-2}=[\mathrm{np} /(1-\mathrm{p})]$ and is therefore independent of $\mathrm{q}$, while $\mathrm{M}=\mathrm{npq}$. When mean postsynaptic potential amplitude changes, no change in $\mathrm{CV}^{-2}$ indicates that only $\mathrm{q}$ has changed and a postsynaptic site is involved. A larger proportional change in $\mathrm{CV}^{-2}$ than in $\mathrm{M}$ indicates that $\mathrm{p}$ has changed, whereas a similar proportional change in the two parameters indicates that $\mathrm{n}$ has changed. Both presynaptic and postsynaptic sites are affected when a smaller proportional change in $\mathrm{CV}^{-2}$ than in $M$ is revealed.

Immunostainings and confocal microscopy. Double transgenic mice (i.e $\mathrm{PV}^{+}$-tdTOM::WT and $\mathrm{PV}^{+}-$ $\left.\mathrm{tdTOM}:: \mathrm{SR}^{-/-}\right)$were anesthetized with intraperitoneal injection of sodium pentobarbital $(60 \mathrm{mg} / \mathrm{kg}$ body weight) and perfused intracardiacally with phosphate buffered saline (PBS 0.1M; pH7.4), followed by freshly dissolved $4 \%$ paraformaldehyde in PBS. The brains were then carefully removed and postfixed overnight in cold fixative. Brain coronal sections ( $25 \mu \mathrm{m}$ thick) were obtained using a vibratome (Leica systems, VT1000S) collected in PBS and incubated for 30 min with PBS containing $0.5 \%$ sodium borohydride for quenching endogeneous fluorescence before blocking and permeabilisation with $0.3 \%$ Triton-X100 and 8\% normal goat serum (NGS) in PBS for $1 \mathrm{~h}$. Brain sections were then stained $48 \mathrm{~h}$ at $4^{\circ} \mathrm{C}$ with the single-domain antibody FluoTag-Q anti-RFP conjugated to AbberiorStar580 (Clone $1 \mathrm{H} 7$; NanoTag Biotechnologies) diluted at $1: 1000$ in PBS containing $0.1 \%$ Tx-100 and 2\% NGS. After five washes in PBS + 0.1\% Tx-100, brain slices were in some case counterstained with DAPI (1:10 000) for 5 min, before further washes and mounted in Vectashield (Vector Labs). Brain sections were examined with an inverted confocal laser-scanning microscope (Leica TCS SP8). Stacks of consecutive confocal images taken with a 10x or a $40 x$ objective were acquired at $651 \mathrm{~nm}$ and $405 \mathrm{~nm}$ sequentially. $Z$ projections were reconstructed using Fiji software.

Enzymes and drugs. The selective depletion of d-serine and glycine was achieved by using recombinant wild-type Rhodotorula gracilis D-amino acid oxidase (RgDAAO, EC 1.4.3.3) and recombinant H244K variant of Bacillus subtilis glycine oxidase (BsGO, EC 1.4.3.19) respectively. Enzymes were overexpressed in Escherichia coli cells, purified and used as described earlier ${ }^{29-31}$. The final RgDAAO and BsGO preparations had a specific activity of approximatively $75 \mathrm{U} / \mathrm{mg}$ protein on d-serine as substrate and 2.5 $\mathrm{U} / \mathrm{mg}$ protein on glycine as substrate, respectively. Slices were first pre-incubated during 45 minutes at $32^{\circ} \mathrm{C}$ with either $R g \mathrm{DAAO}$ or $B s G O$ (both at $0.4 \mathrm{U} / \mathrm{ml}$ ) before recordings. Enzymes were then continuously bath-applied at $0.2 \mathrm{U} / \mathrm{ml}$ during the whole recording.

D-Serine and glycine endogenous levels were elevated using 5-Chloro-benzo[d]isoxazol-3-ol (CBIO, $1 \mu \mathrm{M})$, an inhibitor of DAAO ${ }^{32}$, and N-[3-(4'-Fluorophenyl)-3-(4'-phenylphenoxy)propyl]sarcosine hydrochloride $(A L X 5407,1 \mu \mathrm{M})$, a selective inhibitor of the glycine transporter Gly $11^{28}$, respectively.

ALX5407, D-AP5, MK801 maleate, QX314 bromide, NBQX disodium salt, NMDA and picrotoxin were from either Tocris (Bristol, UK), or HelloBio (Bristol, UK). Strychnine was from Sigma-Aldrich France (SaintQuentin, France). Final concentration of DMSO vehicle in bathing ACSF was kept at 1/4000. 
Statistics. Analysis was conducted using Prism 8 software (GraphPad, SanDiego, California). Results are expressed as mean \pm SEM and a p-value lesser than 0.05 was considered significant. In the figures legends, $\mathrm{n}$ refers to the number of recorded cells sampled from 2-6 mice depending on conditions. Twoway ANOVA with repeated measurements were done for firing frequency, input/output curve, NMDA IV curve and short-term plasticity experiments with post-hoc multiple comparison tests using a BonferroniDunn correction factor when necessary. For time-course experiment and LTP, the mean amplitude of the last 5 minutes of recording (expressed in percent of change compared to control) was tested versus 0 (and versus 100 for LTP) with one-sample Wilcoxon test. Other comparisons were done using the Wilcoxon test for paired data and the Mann-Whitney test for unpaired data.

\section{Declarations}

\section{Acknowledgements}

We thank Zoltan Nüsser (Hungarian Academy of Sciences, Laboratory of Cellular Neurophysiology, Budapest) for valuable discussion and advices during the elaboration of the study, Magalie Martineau, Dominique Debanne (UNIS, INSERM Aix-Marseille University) and Isis Nem Oliveira de Souza (LuMIn, Université Paris-Saclay) for their critical reading and feedback on the manuscript. Michael Rogawski and Sarah Denson (University of California, Davis) provided the SR knock-out mice. Laurent Venance (CIRB, College de France, Paris) and Desdemona Fricker (INCC, University of Paris) provided the Ai9-

tdTomato::PV-Cre transgenic line. The authors also acknowledge the assistance of Ayma Galland, Valérie Domergue and the personnel of the animal facility AnimEX in mouse breeding and care. We thank Maelle Rousselot for her technical assistance with immunostainings and microscopy sessions. This work was supported by the Fondation pour la Recherche Médicale (Equipe FRM DEQ20150331734 to J-PM), Centre National de la Recherche Scientifique (to J-PM and BP), ENS Paris-Saclay (J-PM and BP) and Université Paris-Saclay (to J-PM and BP). LP was supported by a grant form Fondo di Ateneo per la Ricerca.

\section{Author contributions}

J-PM conceptualized and supervised the project. PL and SM with the help of BP carried out and analyzed the electrophysiological experiments. SM and J-PM performed confocal immunohistochemistry. LP provided $R g D A A O$ and BsGO. J-PM wrote the manuscript which was reviewed, edited and approved by all authors.

Competing interests: The authors declare no competing interests.

Data and materials availability: All data are available in the main text or the supplementary materials.

\section{References}

1. Tremblay, R., Lee, S. \& Rudy, B. GABAergic interneurons in the neocortex: From cellular properties to circuits. Neuron 91, 260-292 (2016). 
2. Fishell, G. \& Kepecs, A. Interneuron Types as Attractors and Controllers. Annu. Rev. Neurosci. 43, 1-30 (2020).

3. Hu, H., Gan, J. \& Jonas, P. Interneurons. Fast-spiking, parvalbumin $\bigotimes$ GABAergic interneurons: from cellular design to microcircuit function. Science 345, 1255263 (2014)

4. Sohal, V.S., Zhang, F., Yizhar, O. \& Deisseroth, K. Parvalbumin neurons and gamma rhythms enhance cortical circuit performance. Nature 459, 698-702 (2009)

5. Gonzalez-Burgos, G., Cho, R.Y. \& Lewis, D.A. Alterations in cortical network oscillations and parvalbumin neurons in schizophrenia. Biol. Psychiatry. 77,1031-1040 (2015).

6. Sauer, J.F., Strüber, M. \& Bartos, M. Impaired fast-spiking interneuron function in a genetic mouse model of depression. eLife doi:10.7554/eLife.04979 (2015).

7. Martin, J.Z.D.S. et al. Alterations of specific cortical GABAergic circuits underlie abnormal network activity in a mouse model of Down syndrome. eLife doi:10.7554/eLife.58731 (2020).

8. Carlén, M. et al. A critical role for NMDA receptors in parvalbumin interneurons for gamma rhythm induction and behavior. Mol. Psychiatry 17, 537-548 (2012).

9. Korotkova, T., Fuchs, E.C., Ponomarenko, A., von Engelhardt, J. \& Monyer, H. NMDA receptor ablation on parvalbumin-positive interneurons impairs hippocampal synchrony, spatial representations, and working memory. Neuron 68, 557-569 (2010).

10. Lee, E. et al. Excitatory synapses and gap junctions cooperate to improve Pv neuronal burst firing and cortical social cognition in Shank2-mutant mice. Nat Commun. 12(1):5116 (2021).

11. Nakazawa et al Spatial and temporal boundaries of NMDA receptor hypofunction leading to schizophrenia. NPJ Schizophrenia 3, 7 (2017).

12. Hansen, KB. et al. Structure, Function, and Pharmacology of Glutamate Receptor Ion Channels. Pharmacol Rev. 73(4):298-487 (2021).

13. Paoletti, P., Bellone, C. \& Zhou, Q. NMDA receptor subunit diversity: impact on receptor properties, synaptic plasticity and disease. Nat Rev Neurosci. 14, 383-400 (2013).

14. Mothet, J-P., Le Bail, M. \& Billard, J-M.Time and space profiling of NMDA receptor co-agonist functions. J Neurochem. 135, 210-225 (2015).

15. Johnson, J.W. \& Ascher, P. Glycine potentiates the NMDA response in cultured mouse brain neurons. Nature 325, 529-531 (1987).

16. Kleckner, N.W. \& Dingledine, R. Requirement for glycine in activation of NMDA-receptors expressed in Xenopus oocytes. Science 241, 835-837 (1988). 
17. Mothet, J-P., et al. d-Serine is an endogenous ligand for the glycine site of the N-methyl-D-aspartate receptor. Proc Natl Acad Sci USA 97, 4926-4931 (2000).

18. Anastasiades, P.G. \& Carter, A.G. Circuit organization of the rodent medial prefrontal cortex. Trends Neurosci. 44, 550-563 (2021).

19. Le Merre, P., Ährlund-Richter, S. \& Carlén, M.The mouse prefrontal cortex: unity in diversity. Neuron 109, 1925-1944 (2021).

20. Gao, W.J, et al. Aberrant maturation and connectivity of prefrontal cortex in schizophreniacontribution of NMDA receptor development and hypofunction. Mol Psychiatry. doi: 10.1038/s41380021-01196-w. (2021)

21. Paus, T. et al. Why do many psychiatric disorders emerge during adolescence? Nature Reviews Neurosci. 9, 947-957 (2008).

22. Akgül, G. \& McBain, C.J. Diverse roles for ionotropic glutamate receptors on inhibitory interneurons in developing and adult brain. J Physiol. 594, 5471-5490 (2016).

23. Booker, S.A. \& Wyllie, D.J.A. NMDA receptor function in inhibitory neurons.. Neuropharmacology 196:108609 (2021).

24. Chittajallu, R., et al. Afferent specific role of NMDA receptors for the circuit integration of hippocampal neurogliaform cells. Nat Commun 8,152 (2017).

25. Nevian, T. \& Sakmann, B. Spine $\mathrm{Ca}^{2+}$ signaling in spike-timing-dependent plasticity. J. Neurosci 26, 11001-11013 (2006).

26. Pafundo, D.E., et al. Presynaptic effects of N-methyl-D-aspartate receptors enhance parvalbumin cell-mediated inhibition of pyramidal cells in mouse prefrontal cortex. Biol Psychiatry 84, 460-470 (2018).

27. Wang, H.X. \& Gao, W.J. Cell type-specific development of NMDA receptors in the interneurons of rat prefrontal cortex. Neuropsychopharmacology 34, 2028-2040 (2009).

28. Atkinson, B.N. et al. ALX 5407: a potent, selective inhibitor of the hGlyT1 glycine transporter. Mol. Pharmacol. 60, 1414-1420 (2001)

29. Papouin, T. et al. Synaptic and extrasynaptic NMDA receptors are gated by different endogenous coagonists. Cell 150, 633-646 (2012).

30. Li, Y. et al. Identity of endogenous NMDAR glycine site agonist in amygdala is determined by synaptic activity level. Nat Commun. 4, 1760 (2013). https://doi.org/10.1038/ncomms2779. 
31. Le Bail, M. et al. Identity of the NMDA receptor coagonist is synapse specific and developmentally regulated in the hippocampus. Proc. Natl. Acad.Sci. USA 112, 204-213 (2015).

32. Ferraris $\mathrm{D}$, et al. Synthesis and biological evaluation of D-amino acid oxidase inhibitors. $J$ Med Chem. 51, 3357-3359 (2008).

33. Hashimoto, K. et al. Co-administration of a D-amino acid oxidase inhibitor potentiates the efficacy of D-serine in attenuating prepulse inhibition deficits after administration of dizocilpine. Biol. Psychiatry 65, 1103-1106 (2009).

34. Pollegioni, L. \& Sacchi, S. Metabolism of the neuromodulator d-serine.Cell Mol Life Sci. 67, 23872404 (2010).

35. Miyoshi, Y., et al. Alteration of intrinsic amounts of d-serine in the mice lacking serine racemase and D-amino acid oxidase.Amino Acids 43,1919-1931 (2012).

36. Verrall $\mathrm{L}$, et al. he neurobiology of $\mathrm{D}$-amino acid oxidase and its involvement in schizophrenia. Mol Psychiatry 15, 122-37 (2010).

37. Sasabe, J. et al. Activity of D-amino acid oxidase is widespread in the human central nervous system. Front Synaptic Neurosci. Jun 10;6:14 (2014).

38. Wolosker, H., Blackshaw, S. \& Snyder, S.H. Serine racemase: a glial enzyme synthesizing d-serine to regulate glutamate N-methyl-D-aspartate neurotransmission. Proc Natl Acad Sci USA 96, 13409-13414 (1999).

39. Raboni, S, et al. The Energy Landscape of Human Serine Racemase. Front Mol Biosci. Jan 9;5:112.. (2019).

40. Coyle, JT \& Balu, DT. The Role of Serine Racemase in the Pathophysiology of Brain Disorders. Adv Pharmacol. 82:35-56 (2018)

41. Basu, A.C., et al. Targeted disruption of serine racemase affects glutamatergic neurotransmission and behavior. Mol Psychiatry 14, 719-727 (2009).

42. Balu, D.T. et al. Multiple risk pathways for schizophrenia converge in serine racemase knockout mice, a mouse model of NMDA receptor hypofunction. Proc. Natl. Acad.Sci. USA 110, 2400-2409 (2013).

43. Sullivan, S.J., et al. Serine racemase deletion abolishes light-evoked NMDA receptor currents in retinal ganglion cells. J Physiol. 589, 5997-6006 (2011).

44. Santini, MA. et al. D-serine deficiency attenuates the behavioral and cellular effects induced by the hallucinogenic 5-HT(2A) receptor agonist DOI. Behav Brain Res. 2014 259:242-6 (2014). 
45. Labrie, V. et al. Serine racemase is associated with schizophrenia susceptibility in humans and in a mouse model. Hum Mol Genet. 18(17):3227-43 (2009).

46. Dallerac, G. et al. Dopaminergic neuromodulation of prefrontal cortex activity requires the NMDA receptor coagonist D-serine. Proc Natl Acad Sci USA 118, e2023750118 (2021).

47. Liu, L., et al. Cell type-differential modulation of prefrontal cortical GABAergic interneurons on low gamma rhythm and social interaction. Sci Adv 6(30):eaay4073 (2020).

48. Kawaguchi, Y., et al. Control of excitatory hierarchical circuits by parvalbumin-FS basket cells in layer 5 of the frontal cortex: insights for cortical oscillations. J Neurophysiol. 121, 2222-2236 (2019)

49. Ter Wal, M. \& Tiesinga, P.H.E. Comprehensive characterization of oscillatory signatures in a model circuit with PV- and SOM-expressing interneurons. Biol Cybern. 115, 487-517 (2021).

50. Wang, X.J. Synaptic basis of cortical persistent activity: the importance of NMDA receptors to working memory. J Neurosci. 19, 9587-9603 (1999).

51. Chen C, Blitz DM, Regehr WG. Contributions of receptor desensitization and saturation to plasticity at the retinogeniculate synapse. Neuron 33, 779-788 (2002).

52. Nissen, W., et al. Cell type-specific long-term plasticity at glutamatergic synapses onto hippocampal interneurons expressing either parvalbumin or CB1 cannabinoid receptor. J Neurosci. $\mathbf{3 0 , 1 3 3 7 - 1 3 4 7}$ (2010).

53. Le Roux, N., et al. Input-specific learning rules at excitatory synapses onto hippocampal parvalbumin-expressing interneurons. J Physiol. 591, 1809-1822 (2013).

54. Szegedi, V., et al. Plasticity in single axon glutamatergic connection to GABAergic interneurons regulates complex events in the human neocortex. PLoS Biol. 14, e2000237 (2016).

55. Lau, P.Y., et al. Long-term plasticity in identified hippocampal GABAergic interneurons in the CA1 area in vivo. Brain Struct Funct. 222, 1809-1827 (2017).

56. He, X. et al. Gating of hippocampal rhythms and memory by synaptic plasticity in inhibitory interneurons. Neuron 109, 1013-1028.e9 (2021).

57. Capogna, M. et al. The ins and outs of inhibitory synaptic plasticity: Neuron types, molecular mechanisms and functional roles. Eur J Neurosci. 54, 6882-6901 (2021).

58. Aguilar, DD. et al. Altered neural oscillations and behavior in a genetic mouse model of NMDA receptor hypofunction. Sci Rep. 11, 9031.(2021).

59. Balla, A. et al. Translational neurophysiological biomarkers of N-methyl-d-aspartate receptor dysfunction in serine racemase knockout mice. Biomark Neuropsychiatry. Jun;2:100019 (2020). 
60. Ploux, E. et al. Serine racemase deletion affects the excitatory/inhibitory balance of the hippocampal CA1 network. Int J Mol Sci. 21, 9447. (2020). https://doi.org/10.3390/ijms21249447

61. Jami, S.A. et al. Increased excitation-inhibition balance and loss of GABAergic synapses in the serine racemase knockout model of NMDA receptor hypofunction. J Neurophysiol. 126, 11-27 (2021).

62. Miyamae, T., et al. Distinct Physiological Maturation of parvalbumin-positive neuron subtypes in mouse prefrontal cortex. J. Neurosci. 37, 4883-4902 (2017)

63. Henneberger, $\mathrm{C}$. et al. Long-term potentiation depends on release of D-serine from astrocytes. Nature 463,232-236 (2010).

64. Fossat, P. et al. Glial D-serine gates NMDA receptors at excitatory synapses in prefrontal cortex. Cereb Cortex 22, 595-606 (2012).

65. Rosenberg, D. et al. Neuronal D-serine and glycine release via the Asc- 1 transporter regulates NMDA receptor-dependent synaptic activity. J Neurosci. 33, 3533-44 (2013).,

66. Curcio, L. et al. Reduced D-serine levels in the nucleus accumbens of cocaine-treated rats hinder the induction of NMDA receptor-dependent synaptic plasticity. Brain 136, 1216-30 (2013).

67. Wong, J.M. et al. Postsynaptic Serine Racemase Regulates NMDA Receptor Function.. J Neurosci. 40, 9564-9575 (2020).

68. Li, Y. et al. Glycine site of NMDA receptor serves as a spatiotemporal detector of synaptic activity patterns. J Neurophysiol. 102, 578-589 (2009).

69. Yao, L. et al. Higher ambient synaptic glutamate at inhibitory versus excitatory neurons differentially impacts NMDA receptor activity. Nat Commun. 9, 4000 (2018).

70. Semyanov, A \& Verkhratsky, A. Astrocytic processes: from tripartite synapses to the active milieu. Trends Neurosci. 44, 781-792 (2021).

71. Refaeli, $R$ et al. Features of hippocampal astrocytic domains and their spatial relation to excitatory and inhibitory neurons. Glia 69, 2378-2390 (2021).

72. Pei, J.C. et al. Directly and indirectly targeting the glycine modulatory site to modulate NMDA receptor function to address unmet medical needs of patients with schizophrenia. Front Psychiatry 12, 742058 (2021).

73. Kaiser, T. et al. Transgenic labeling of parvalbumin-expressing neurons with tdTomato. Neuroscience 321, 236-245 (2016).

74. Grover, LM. Evidence for postsynaptic induction and expression of NMDA receptor independent LTP. J Neurophysiol. 79, 1167-1182 (1998). 
A

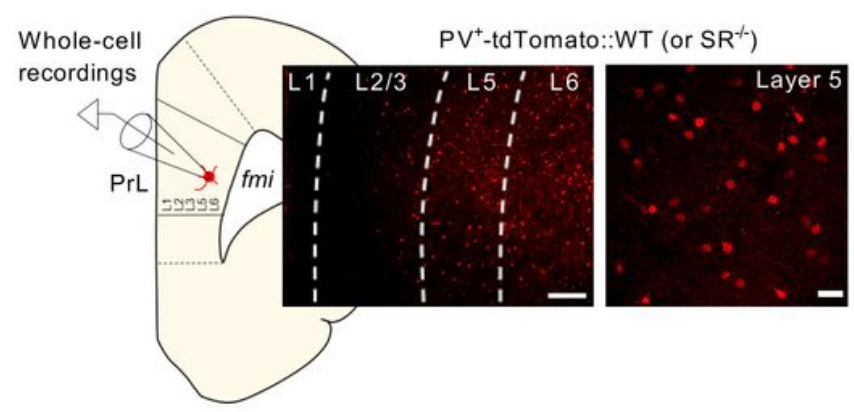

C
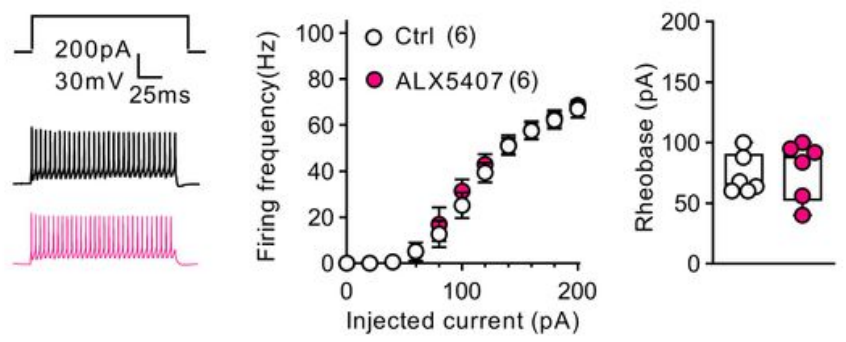

E

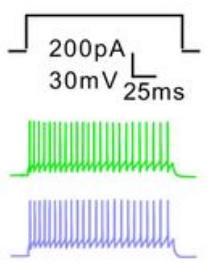

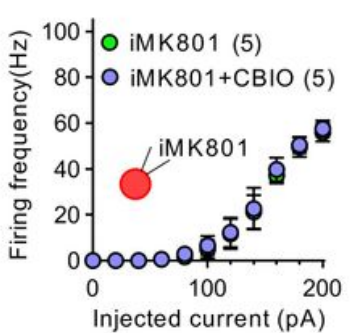

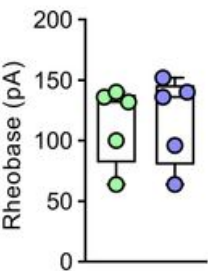

No agonist application
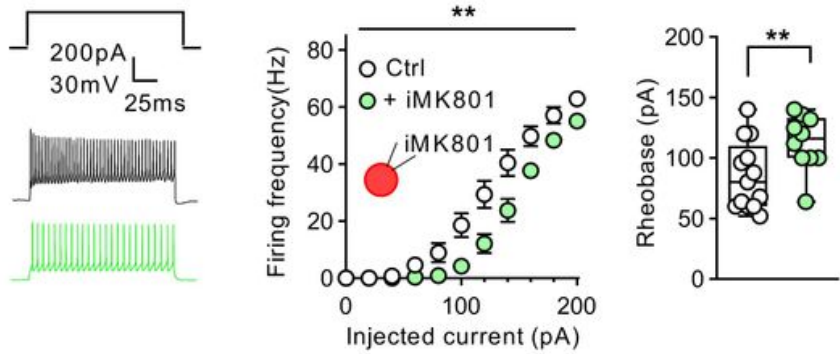

D
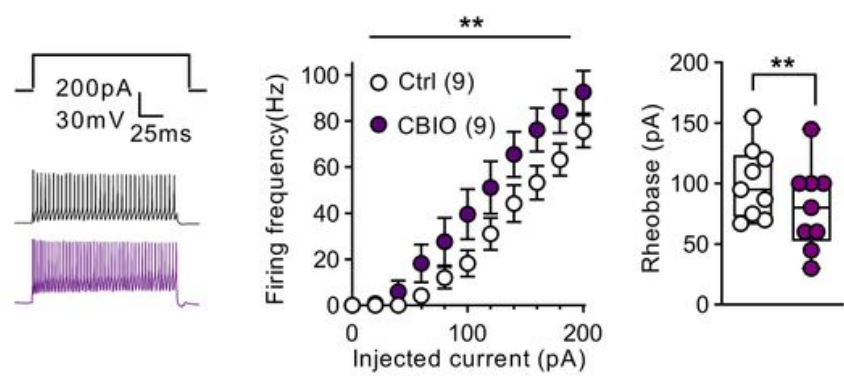

F

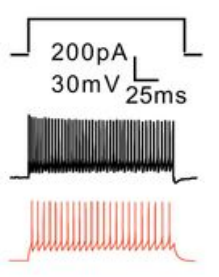

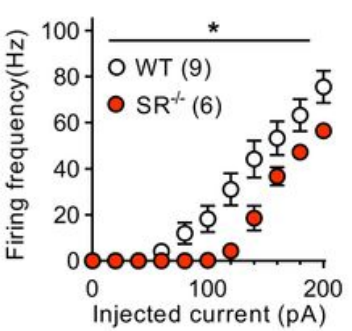

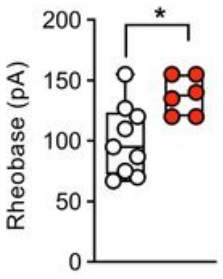

Figure 1

NMDAR co-agonist site occupancy tunes the intrinsic excitability of $\mathrm{PV}^{+}$interneurons. a Schematic diagram depicting the mPFC slice from which layer $\mathrm{V} F S-\mathrm{PV}^{+}$interneurons were recorded in $\mathrm{PV}^{+}-$ tdTomato::WT or $\mathrm{PV}^{+}$-tdTomato::SR ${ }^{-/-}$mice. Representative confocal images of tdTomato expressing neurons in the PrL of P50 mouse across PrL layers (left panel, scale bar: $200 \mu \mathrm{m}$ ) and in layer V (right panel, scale bar: $40 \mu \mathrm{m})$. b Blocking NMDAR by inclusion of MK801 in the patch pipette (iMK801, light green) reduced the firing activity of FS-PV ${ }^{+}$interneurons in WT mice $\left(n=10\right.$ cells; $P=0.0118, \mathrm{~F}_{(1,21)}=7.603$ two-way ANOVA) compared to control ( $n=13$ cells). Right, boxplot graphs show a marked increase of the rheobase with iMK801 compared with Ctrl ( $P=0.0180$, Mann-Whitney test). c Elevation of the glycine levels by blockade of GlyT1 with ALX5407 (fuschia) affected neither the firing pattern of the neurons ( $n=$ 6 cells for ALX5407 and for Ctrl; $P=0.6617, F_{(1,5)}=0.2159$ two-way ANOVA) or the rheobase (right boxplots, $P=0.8750$, Wilcoxon test). $\mathbf{d}$ Elevation of $\mathrm{d}$-serine levels through the application of CBIO 
(purple) to blunt DAAO activity increased the firing rate ( $n=9$ cells for $\mathrm{CBIO}$ and for Ctrl; $P=0.0123$, $\mathrm{F}_{(1,8)}=10.34$ two-way ANOVA) by reducing the rheobase (right boxplots; $P=0.0039$, Wilcoxon test). $\mathbf{e}$ iMK801 nulled the promoting action of CBIO on firing frequency ( $n=5$ cells for $\mathrm{CBIO}$ and for Ctrl; $P=$ $0.2094, F_{(1,4)}=2.233$ two-way ANOVA). Boxplot graphs show that the rheobase was no longer modified by CBIO upon block of NMDAR ( $P=0.7381$, Mann-Whitney test). ${\mathrm{f} \mathrm{FS}-\mathrm{PV}^{+} \text {interneurons from SR}}^{-/-}$mice (dark orange, $n=6$ cells) showed a marked reduction in their firing activity compared to WT mice ( $n=9$ cells) $\left(P=0.2097, \mathrm{~F}_{(1,13)}=5.958\right.$ two-way ANOVA $)$ that is associated with an increase of the rheobase $(P=$ 0.0244 , Mann-Whitney test) thus phenocopying the effect of iMK801. 
A
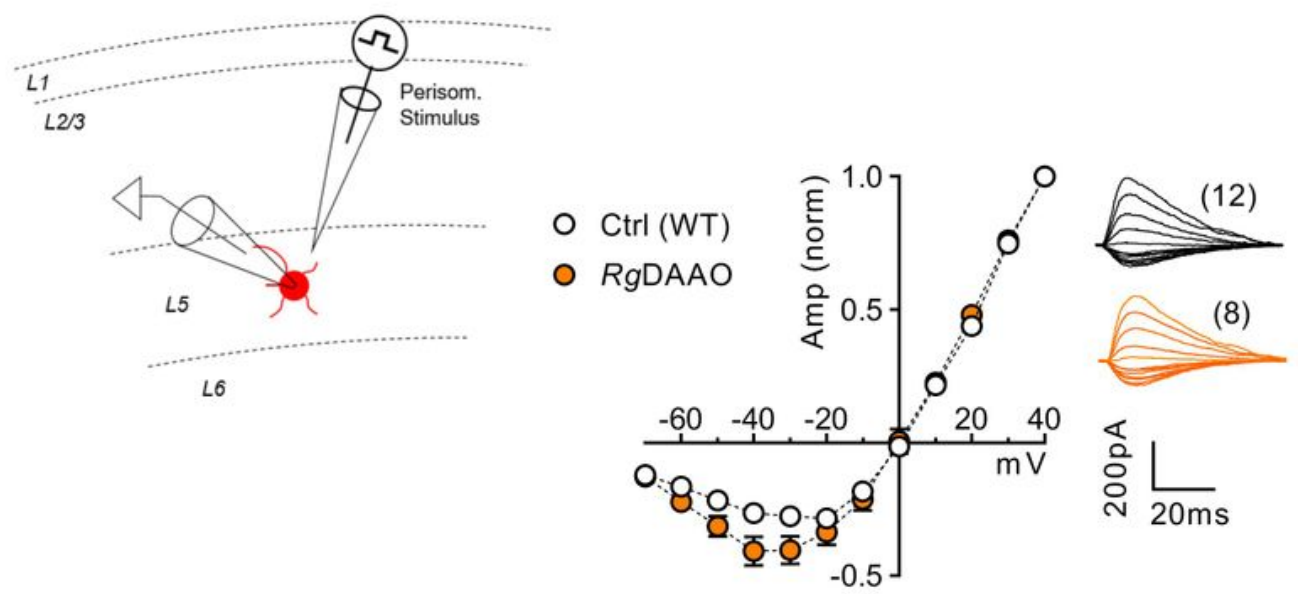

B

@ $0.1 \mathrm{~Hz}$

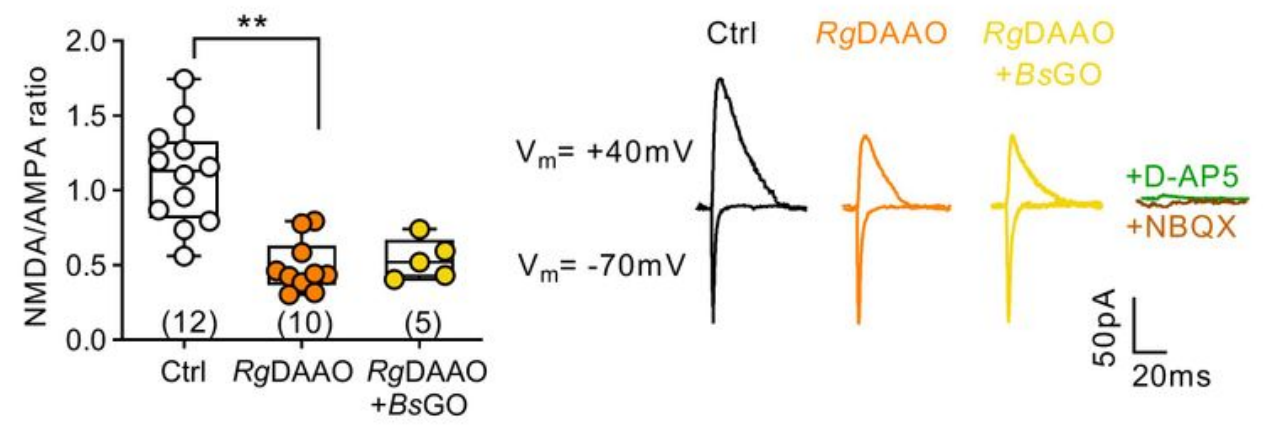

C

@ $0.1 \mathrm{~Hz}$
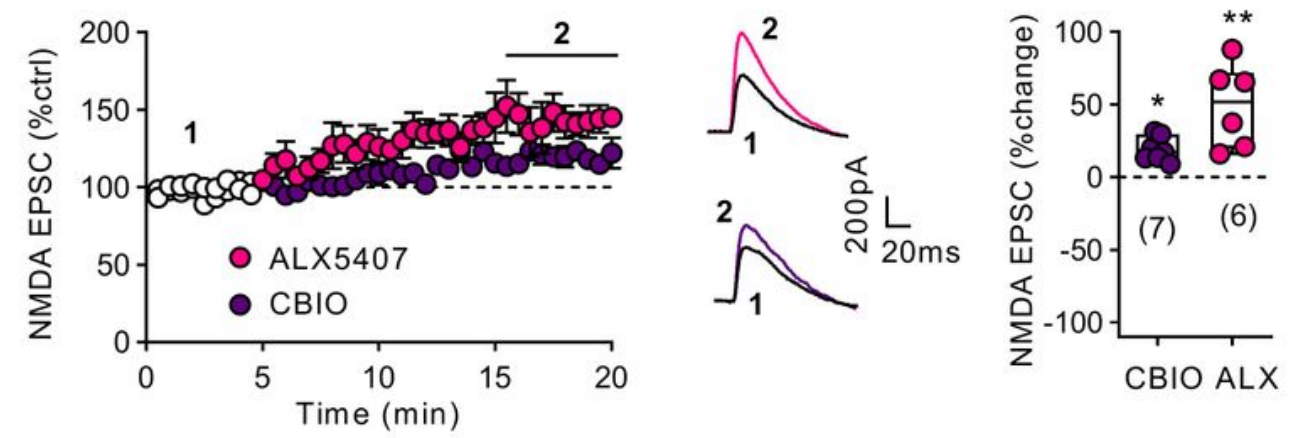

Figure 2

Strength of excitatory input onto FS-PV ${ }^{+}$GABAergic neurons obeys unrequitedly to d-serine co-agonism. a Left, the schematic diagram shows the experimental configurations for recording the isolated evoked excitatory postsynaptic currents (EPSCs) from tdTomato fluorescent FS-PV ${ }^{+}$cells in layer 5 of mPFC. EPSCs were elicited in response to focal extracellular perisomatic stimulation. Right, current-voltage (I-V) relationship of normalized NMDA-EPSCs in WT mice ( $n=12$ cells, white circles and black traces) was 
unaltered by treating the slices with $\operatorname{RgDAAO}$ ( $n=8$, orange circles and traces) that acutely depletes dserine $\left(P=0.1341, \mathrm{~F}_{(1,17)}=2,474\right.$ two-way ANOVA). Representative traces of NMDA-EPSCs recorded from $-70 \mathrm{mV}$ to $+40 \mathrm{mV}$ for control and RgDAAO conditions are illustrated. b NMDA-EPSCs (+40 mV) and AMPA-EPSCs $(-70 \mathrm{mV})$ were abolished in the presence of D-AP5 (green trace, $n=4$ cells) and NBQX (brown trace, $n=4$ cells) respectively. Treatment of WT slices with RgDAAO (orange circles and traces) markedly reduced NMDAR/AMPAR ratio by $\sim 50 \%$ from $1.10 \pm 0.10$ ( $n=12$ cells) to $0.49 \pm 0.05(n=10$ cells) but concurrent application of $B s G O$ (yellow symbols and traces) showed no further effect $(0.54 \pm$ $0.06, n=5$ cells) ( $P=0.0015$, Kruskal-Wallis test; Ctrl vs RgDAAO: $P=0.0018, R g D A A O$ vs BsGO: $P>$ 0.9999, post-hoc Dunn's test). c Time course shows that bath application of CBIO (purple symbols and traces) on WT slices to promote the synaptic availability of d-serine increased NMDA-EPSCs by $22.16 \pm$ $4.58 \%$ ( $n=7$ cells: $P=0.0156$, Wilcoxon test). Bath application of ALX5407 (fuschia) to increase the synaptic availability of glycine potentiated EPSCs $(n=6$ cells; $+49.23 \pm 11.69 \%, P=0.0313$, Wilcoxon test). The greatest increase of the NMDA-EPSCs in ALX5407 than in CBIO likely reflected that GlyT1 normally prevent glycine access to synaptic NMDARs. 
A
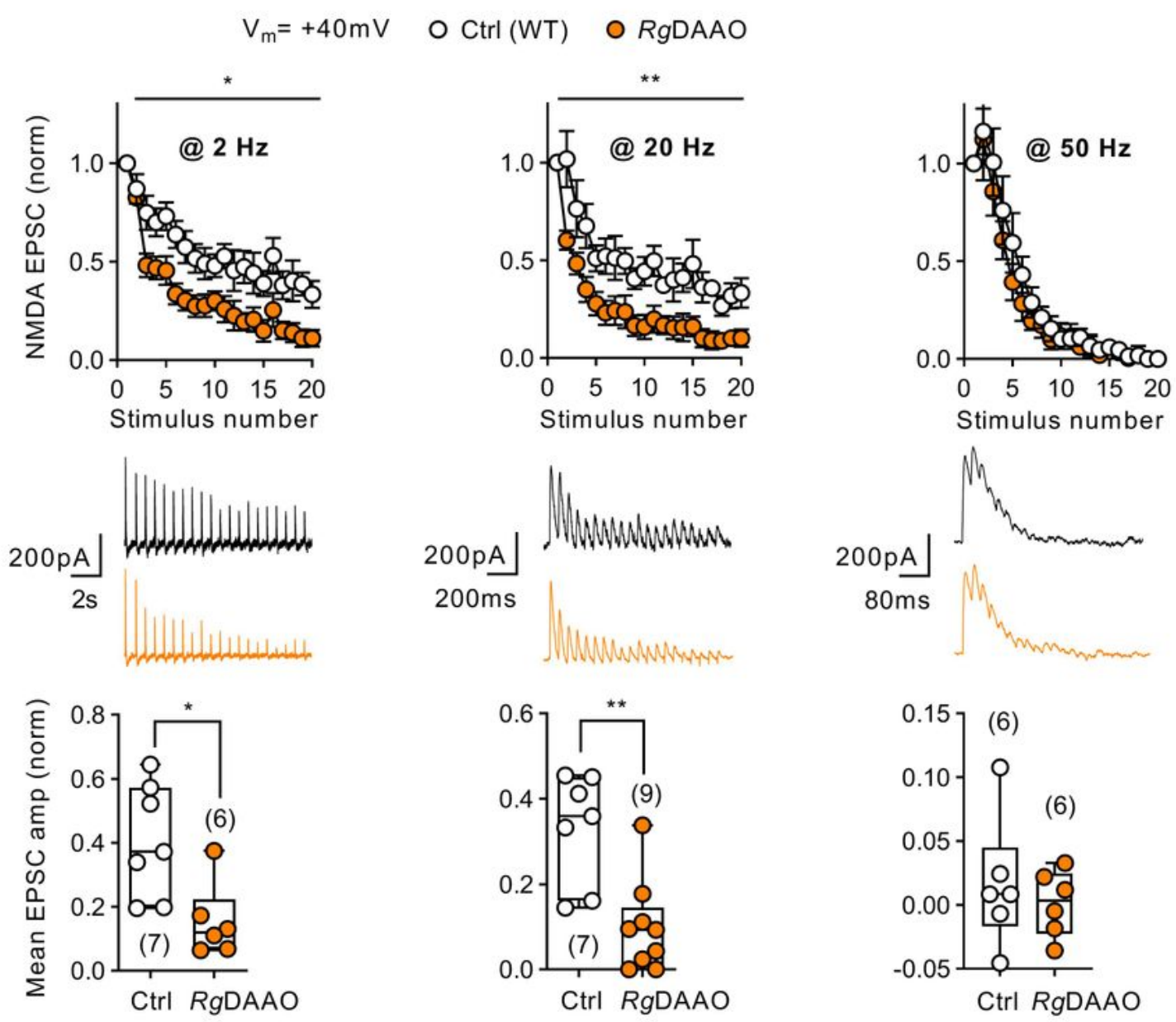

B
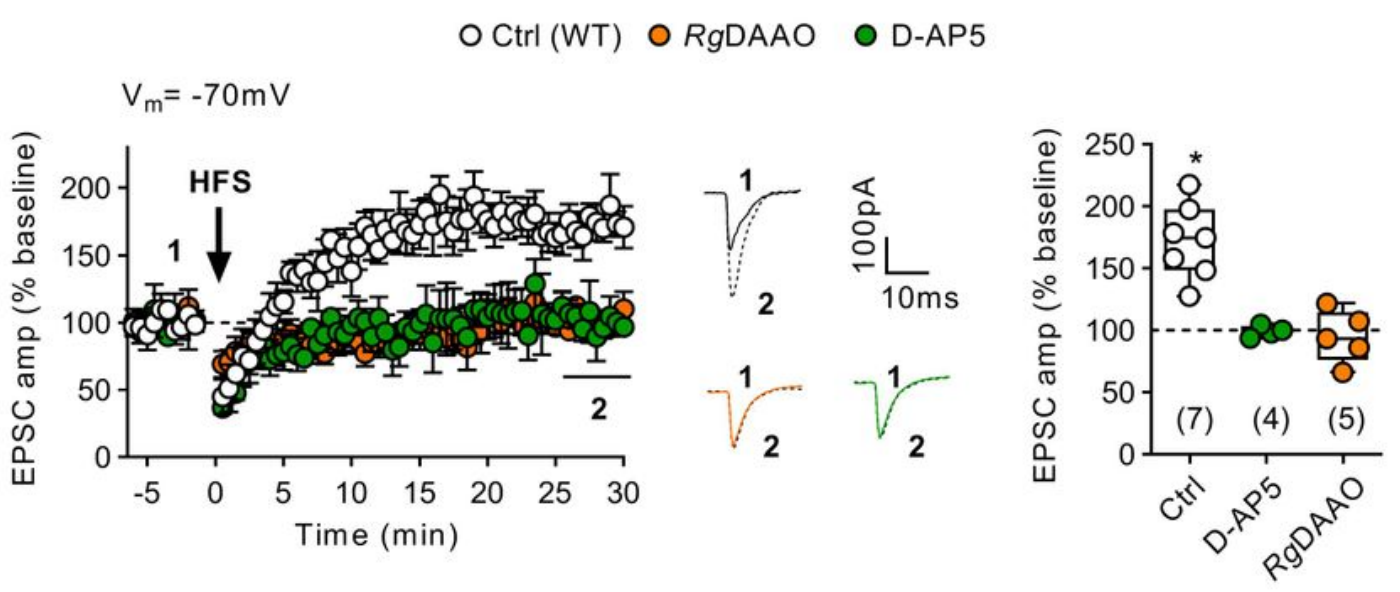

\section{Figure 3}

\section{d-serine co-agonism enables synaptic plasticity of PC excitatory output synapse onto FS-PV ${ }^{+}$}

interneurons. a NMDAR co-agonist and PC-to-PV synapse adaptation to increase synaptic regime.

Normalized EPSCs amplitudes in response to stimulation trains applied at increasing frequencies $(2,20$ and $50 \mathrm{~Hz}$ ). As the frequency increases, control EPSCs showed graded attenuation (Upper graphs: white circles and middle black traces). At $2 \mathrm{~Hz}$, acute d-serine depletion (RgDAAO, orange traces and circles) 
increased short-term depression ( $n=7$ cells for Ctrl vs $n=6$ cells for RgDAAO; $P=0.0107, \mathrm{~F}_{(1,11)}=2,474$ two-way ANOVA). At $20 \mathrm{~Hz}$, treating the WT slices with RgDAAO had a more accentuated effect on shortterm depression ( $n=7$ cells for Ctrl vs $n=9$ cells for $\operatorname{RgDAAO} P=0.0037, \mathrm{~F}_{(1,14)}=12.05$ two-way ANOVA). At $50 \mathrm{~Hz}$, no difference was observed between Ctrl ( $n=6$ cells) and RgDAAO ( $n=6$ cells) groups ( $P=$ $0.4497, \mathrm{~F}_{(1,10)}=0.6188$ two-way ANOVA) likely reflecting that the action of the co-agonist in this form of short-term plasticity is limited to frequencies below $50 \mathrm{~Hz}$. Boxplot graphs (Bottom) show the comparison of the mean normalized NMDA-EPSCs amplitude in Ctrl and RgDAAO for the 5 last points at $2 \mathrm{~Hz}(P=$ 0.0140 , Mann-Whitney test), at $20 \mathrm{~Hz}(P=0.0033$, Mann-Whitney test) and $50 \mathrm{~Hz}(P=0.9372$, MannWhitney test). $\mathbf{b}$ Excitatory long term potentiation (LTP) at the PC-to-PV synapse is D-AP5 sensitive and dependent on d-serine co-agonism. Left panel shows the time-course of LTP induced by repeated $100 \mathrm{~Hz}$ tetanus stimulation (arrow) in control, D-AP5 or RgDAAO group. Representative AMPA EPSCs are illustrated before (1, continuous lines) and after stimulation (2, dashed lines) in control (black traces), DAP5 (green) and RgDAAO (orange) groups. As shown in right panel the mean normalized amplitude of the 5 last minutes for each group indicated that LTP normally induced in control ( $n=7$ cells, $172.0 \pm 1.44 \%$, $P=0.0156$, Wilcoxon test $v s 100)$ is absent in D-AP5 $(n=4$ cells, $99.4 \pm 2.26 \%, P=0.8750$, Wilcoxon test vs 100) or in RgDAAO ( $n=5$ cells, $95.1 \pm 9.43 \%, P=0.8125$, Wilcoxon test $v s 100$ ) treated slices. Stars represent statistically significant differences. Data are expressed as mean \pm SEM.

A

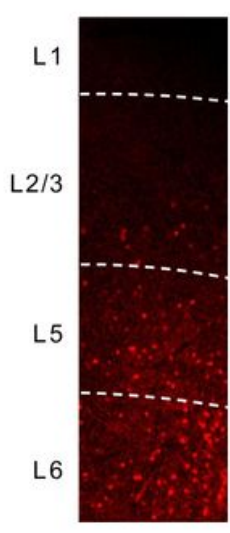

tdTomato

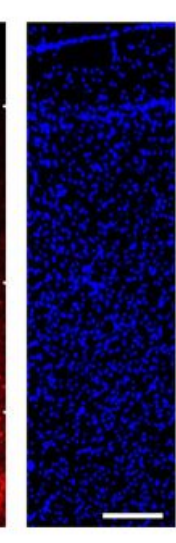

DAPI
B

@ $0.1 \mathrm{~Hz}$

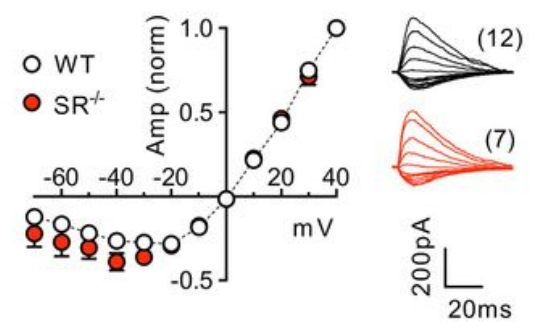

D

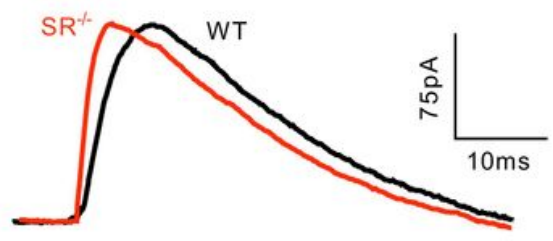

C
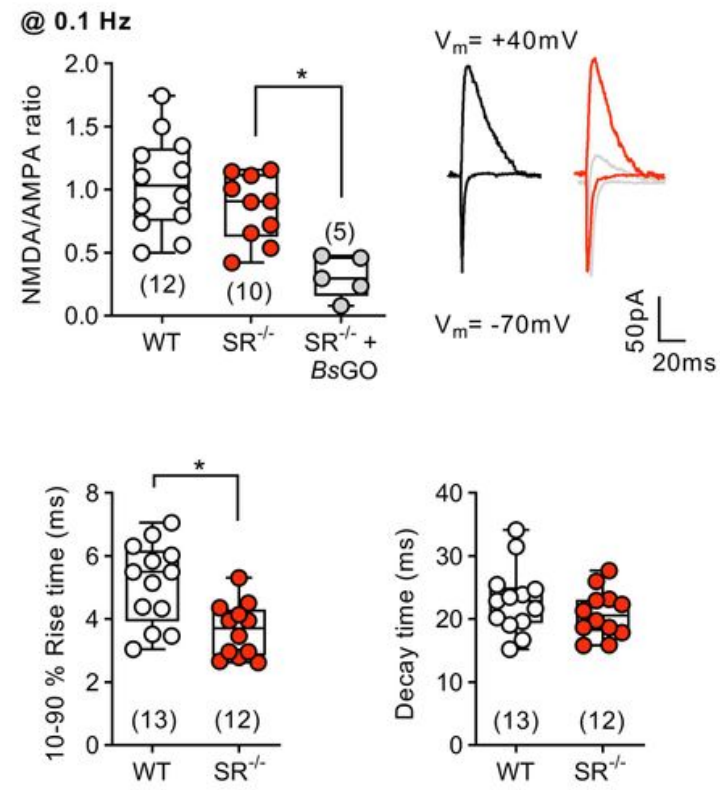

\section{Figure 4}

\section{Glycine is a spare substitute to $\mathrm{d}$-serine as a co-agonist for NMDARs at $\mathrm{PV}^{+}$interneurons at low synaptic} regimes. a Representative confocal image of tdTomato expressing interneurons in the $\mathrm{mPFC}$ of $\mathrm{SR}^{-/-}$ mice. DAPI staining indicates normal lamination and cell density in respect to WT mice. b Currentvoltage (I-V) relationship of normalized NMDA-EPSCs in d-serine-deficient mice ( $n=7$ cells, orange circles 
and traces) was normal in respect to WT mice $(n=12)(P=0.2984, \mathrm{~F}(2,23)=1,275$ two-way ANOVA). Representative traces of NMDA-EPSCs recorded from $-70 \mathrm{mV}$ to $+40 \mathrm{mV}$ for WT and $\mathrm{SR}^{-/-}$mice are illustrated. $\mathbf{c}$ Loss of $d$-serine function in $\mathrm{SR}^{-/-}$mice did not affect NMDAR/AMPAR ratio (WT: $1.10 \pm 0.10$, $n=12$ cells $v s \mathrm{SR}^{-/}$slices: $1.02 \pm 0.10, n=10$ cells) but depletion of glycine with the enzymatic scavenger BsGO significantly reduced it by $\sim 60 \%$ compared to WT slices (SR ${ }^{-/-}+B s G 0,0.37 \pm 0.09, n=5$ cells) $(P=$ 0.0072 , Kruskal-Wallis test; post-hoc Dunn's test, $P>0.9999$ for WT $v s \mathrm{SR}^{-/}, P=0.0085$ for WT $v s \mathrm{SR}^{-/-}+$ $B s G O, P=0.0155$ for $\left.\mathrm{SR}^{-/-} v s \mathrm{SR}^{-/}+B s \mathrm{~B} O\right)$. Representative traces of AMPA-EPSCs and NMDA-EPSCs for control (black), $\mathrm{SR}^{-/-}$(dark orange) and $\mathrm{SR}^{-/}+B S G O$ (light grey) conditions are illustrated. $\mathbf{d}$ Analysis of NMDA-EPSCs activation and deactivation constants in wild-type (black trace) and $\mathrm{SR}^{-/-}$(coral orange). Rise time constant is reduced in NMDA-EPSCs from $\mathrm{SR}^{-/-}$mice (Mann-Whitney test, ${ }^{*} \mathrm{p}=0.0030, \mathrm{n}=13$ and $\mathrm{n}=12$ for $\mathrm{WT}$ and $\mathrm{SR}^{-/}$, respectively) while decay time constant remained the same (Mann-Whitney test, $\mathrm{p}=0.3203, \mathrm{n}=13$ and $\mathrm{n}=12$ for $\mathrm{WT}$ and $\mathrm{SR}^{-/}$, respectively). 
@ 2 Hz
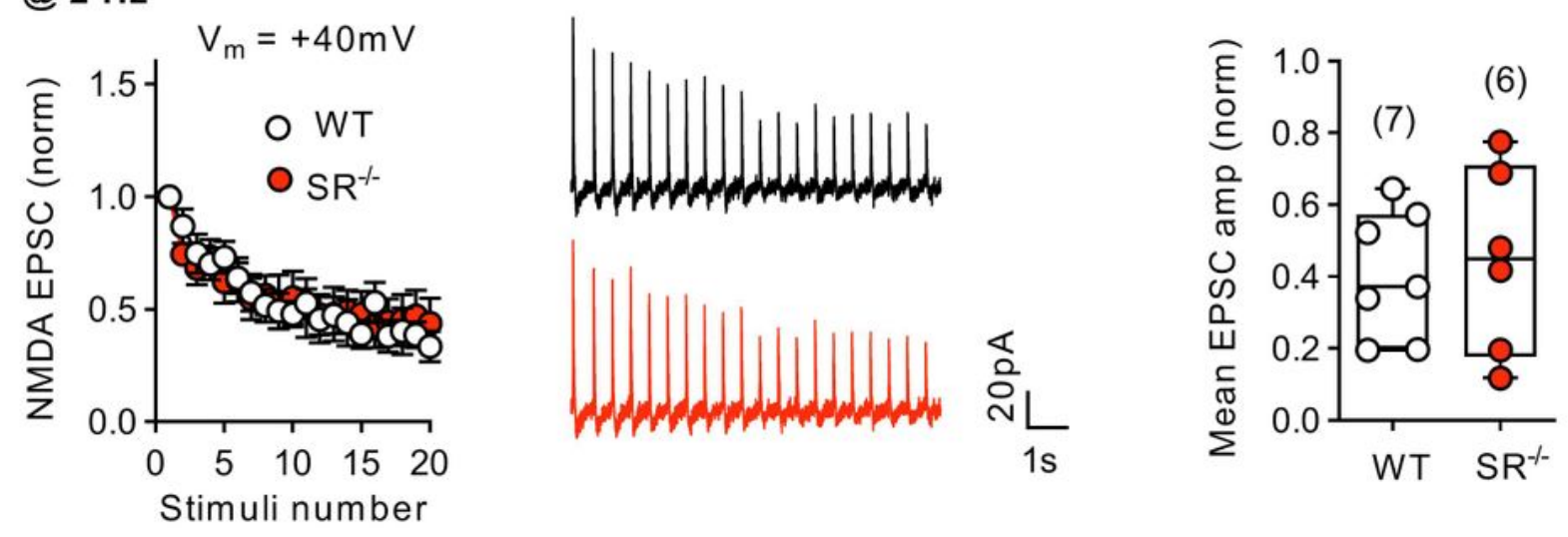

@ $20 \mathrm{~Hz}$
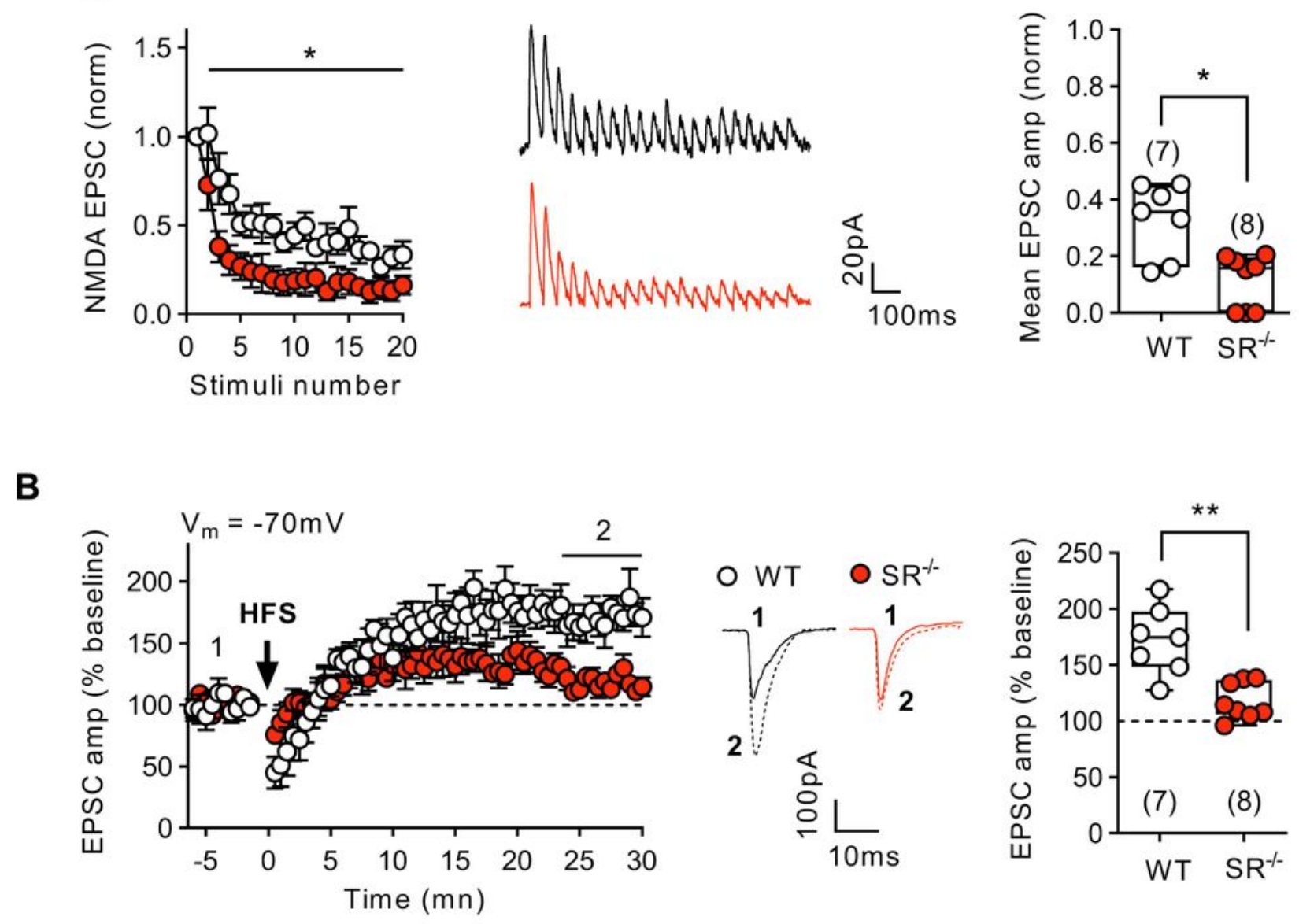

Figure 5

Glycine does not support synaptic plasticity of $\mathrm{PC}$ to $\mathrm{PV}^{+}$interneurons in $\mathrm{SR}^{-/}$mice. a Contribution of glycine to short-term depression is marginal. At $2 \mathrm{~Hz}$, short-term depression in $\mathrm{SR}^{-/-}$( $n=6$ cells) was normal compared to WT slices ( $n=7$ cells) (P=0,9197, $\mathrm{F}(1,11)=0,0106$ two-way ANOVA). In contrast at $20 \mathrm{~Hz}$, chronic deficiency in d-serine impaired short-term plasticity by reinforcing short-term depression (WT: $n=7$ cells; SR ${ }^{--}: n=8$ cells; $P=0,0198, F(1,13)=7,047$ two-way ANOVA) on line with the effect of 
RgDAAO (see Fig 3, $\mathrm{SR}^{-/} v s$ RgDAAO $P>0.9999$, Post-hoc Bonferroni's test after two-way ANOVA). Comparison of the mean normalized NMDA-EPSCs amplitude of the 5 last points at 2 and $20 \mathrm{~Hz}$ for SR ${ }^{-/}$ and control groups reveals no difference at $2 \mathrm{~Hz}(\mathrm{P}=0,9452$, Mann-Whitney test). On the contrary, mean amplitude at $20 \mathrm{~Hz}$ is significantly reduced for $\mathrm{SR}^{-/-}$compared to control $(\mathrm{P}=0,0284$, Mann-Whitney test). $\mathbf{b}$ Long term potentiation (LTP) recorded at the PC-to-PV synapse in $\mathrm{SR}^{-/-}$mice. Time-course of LTP induced by a repeated tetanus (arrow) in WT was significantly reduced in $\mathrm{SR}^{-/-}$mice $(172.0 \pm 1.44 \%$ in WT vs $118.1 \pm 5.80 \%$ in SR ${ }^{-/}$slices; $n=7$ and $n=8$ cells respectively). However, the short-term potentiation 10 min post stimulation was observed in both groups. Mean amplitude after potentiation is reduced in $\mathrm{SR}^{-/}$ groups compared to control (Mann-Whitney test, ${ }^{* \star} p=0.0022$ ).

\section{Supplementary Files}

This is a list of supplementary files associated with this preprint. Click to download.

- LecoufletNatureCommSI.docx 